\title{
The upgraded TOMAS device: A toroidal plasma facility for wall conditioning, plasma production, and plasma-surface interaction studies
}

Cite as: Rev. Sci. Instrum. 92, 023506 (2021); https://doi.org/10.1063/5.0033229

Submitted: 14 October 2020 . Accepted: 12 January 2021 . Published Online: 16 February 2021

(iD) A. Goriaev, (i) T. Wauters, (D) s. Möller, (D) R. Brakel, (D) s. Brezinsek, (iD) J. Buermans, (D) K. Crombé, (iD) A. Dinklage, R. Habrichs, D. Höschen, M. Krause, (D) Yu. Kovtun, (D) D. López-Rodríguez, (D) F. Louche, (D) S. Moon, (D) D. Nicolai, J. Thomas, (D) R. Ragona, (D) M. Rubel, T. Rüttgers, (D) P. Petersson, (D) P. Brunsell, (D) Ch. Linsmeier, and M. Van Schoor

\section{ARTICLES YOU MAY BE INTERESTED IN}

Machine learning prediction of electron density and temperature from He I line ratios Review of Scientific Instruments 92, 023505 (2021); https://doi.org/10.1063/5.0028000

Long-pulse diagnostic calorimeter for the negative ion source testbed BATMAN upgrade Review of Scientific Instruments 92, 023504 (2021); https://doi.org/10.1063/5.0022465

Design and characterization of a resonant microwave cavity as a diagnostic for ultracold plasmas

Review of Scientific Instruments 92, 013506 (2021); https://doi.org/10.1063/5.0037846

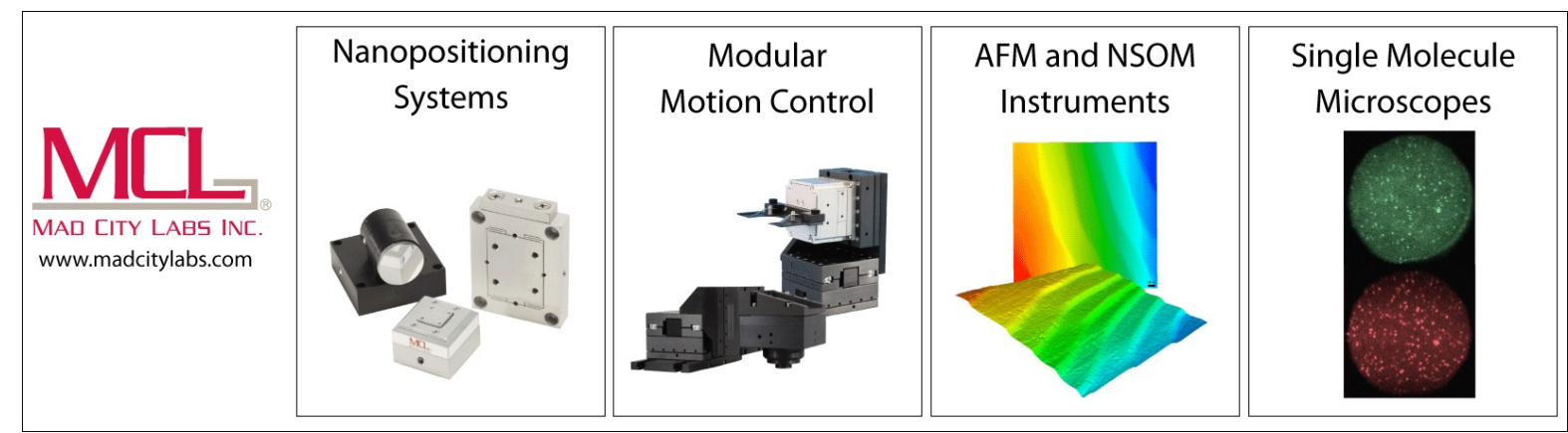




\title{
The upgraded TOMAS device: A toroidal plasma facility for wall conditioning, plasma production, and plasma-surface interaction studies
}

Cite as: Rev. Sci. Instrum. 92, 023506 (2021); doi: 10.1063/5.0033229

Submitted: 14 October 2020 - Accepted: 12 January 2021 •

Published Online: 16 February 2021

A. Goriaev, ${ }^{1,2, a)}$ (D) T. Wauters, ${ }^{1}$ (D) S. Möller, ${ }^{3}$ (D) R. Brakel, ${ }^{4}$ (D) S. Brezinsek, ${ }^{3}$ (D) J. Buermans, ${ }^{1,2}$ (D) K. Crombé,
A. Dinklage,
R. Habrichs
${ }^{3}$ D. Höschen, ${ }^{3}$ M. Krause, ${ }^{4}$ Yu. Kovtun, ${ }^{5}$
D. López-Rodríguez, ${ }^{2}$
F. Louche,

S. Moon, ${ }^{6}$ (D) D. Nicolai, ${ }^{3}$ (D) J. Thomas, ${ }^{3}$ R. Ragona, ${ }^{1}$ (D) M. Rubel, ${ }^{6}$ (D) T. Rüttgers, ${ }^{3}$ P. Petersson, ${ }^{6}$

P. Brunsell, ${ }^{6}$ (D) Ch. Linsmeier, ${ }^{3}$ (D) and M. Van Schoor

\section{AFFILIATIONS}

${ }^{1}$ Laboratory for Plasma Physics, LPP-ERM/KMS, Trilateral Euregio Cluster (TEC) Partner, Brussels, Belgium

${ }^{2}$ Department of Applied Physics, Ghent University, Ghent, Belgium

${ }^{3}$ Institute for Energy and Climate Research—Plasma Physics, Forschungszentrum Jülich $\mathrm{GmbH}$, Jülich, Germany

"Max-Planck-Institute for Plasma Physics, Greifswald, Germany

${ }^{5}$ Institute of Plasma Physics, NSC KIPT, Kharkov, Ukraine

${ }^{6}$ Royal Institute of Technology (KTH), Stockholm, Sweden

a) Author to whom correspondence should be addressed: a.goriaev@fz-juelich.de

\begin{abstract}
The Toroidal Magnetized System device has been significantly upgraded to enable development of various wall conditioning techniques, including methods based on ion and electron cyclotron (IC/EC) range of frequency plasmas, and to complement plasma-wall interaction research in tokamaks and stellarators. The toroidal magnetic field generated by 16 coils can reach its maximum of $125 \mathrm{mT}$ on the toroidal axis. The EC system is operated at $2.45 \mathrm{GHz}$ with up to $6 \mathrm{~kW}$ forward power. The IC system can couple up to $6 \mathrm{~kW}$ in the frequency range of $10 \mathrm{MHz}-50 \mathrm{MHz}$. The direct current glow discharge system is based on a graphite anode with a maximum voltage of $1.5 \mathrm{kV}$ and a current of $6 \mathrm{~A}$. A load-lock system with a vertical manipulator allows exposure of material samples. A number of diagnostics have been installed: singleand triple-pin Langmuir probes for radial plasma profiles, a time-of-flight neutral particle analyzer capable of detecting neutrals in the energy range of $10 \mathrm{eV}-1000 \mathrm{eV}$, and a quadrupole mass spectrometer and video systems for plasma imaging. The majority of systems and diagnostics are controlled by the Siemens SIMATIC S7 system, which also provides safety interlocks.
\end{abstract}

Published under license by AIP Publishing. https://doi.org/10.1063/5.0033229

\section{INTRODUCTION}

Plasma-surface interactions (PSIs) in controlled fusion devices lead to significant surface modifications at the plasma-facing components (PFCs) and concomitant contamination of the fusion plasma by species released from the wall due to sputtering, melting, or evaporation. ${ }^{1,2}$ The wall released impurities, often volatile neutrals, are partially evacuated from the chamber. Nevertheless, most of them are instantly ionized and transported along the magnetic field lines in the plasma edge until they get re-deposited at another surface location. Co-deposition of various impurities present in the plasma occurs together with fuel atoms, leading to the formation of mixed-material layers whose composition and properties distinctly differ from those of the PFC materials. The fuel content in such layers is of particular importance especially in the case of operation with radioactive tritium. ${ }^{1-3}$ To ensure and provide proper wall conditions for plasma operation or to control fuel inventory, a number of wall conditioning techniques have been developed: deposition of thin low-Z films by plasma-assisted procedures or evaporation, baking, glow discharge cleaning (GDC), and, recently, operations 
involving ion or electron cyclotron plasmas. ${ }^{4}$ The two latter methods are being elaborated for use under strong magnetic fields in devices with super conducting magnets, i.e., the stellarator Wendelstein 7-X (W7-X) ${ }^{5}$ and ITER. ${ }^{6}$ Experiments carried out in present fusion devices have proven the usefulness of the techniques, ${ }^{4}$ but due to the limited availability of adapted diagnostics, the complex multi-physics picture of the wall conditioning discharges such as PSI aspects could not yet be fully resolved. Development of these conditioning procedures requires hundreds of tests under a large number of conditions, e.g., antenna settings, gas composition, pressure, magnetic field, etc., for which experimental time and flexibility are essential. This calls for a device dedicated to that specific research program. To this end, this paper presents the upgraded TOroidal MAgnetized System (TOMAS), which has become fully equipped for wall conditioning, plasma production, and PSI studies. The technical capabilities and enhancements resulting in reliable plasma performance and diagnosis are discussed.

\section{THE TOMAS DEVICE}

The TOMAS (originally TOroidal MAgnetized System) device was developed and built at the Kurchatov Insitute in Moscow, Russia, in 1989-1990. Later on, it was exploited by the Forschungszentrum Jülich, Germany, for systematic investigations of electron cyclotron resonance plasmas in a toroidal geometry for wall conditioning. The first description of the facility and the following operational experience related to film deposition were reported in 2000. In the following years, TOMAS was also used in a number of studies related to wall conditioning and plasma surface interaction, i.e., Electron Cyclotron Wall Conditioning $(\mathrm{ECWC})^{8}$ and fuel removal by GDC. ${ }^{9,10}$

TOMAS is constructed as a quasi toroidal vacuum vessel made of stainless steel (wall thickness of $5 \mathrm{~mm}$ ). The vacuum vessel consists of 4 sectors with a minor radius (a radius of the cylindrical sections) of $260 \mathrm{~mm}$. The individual sectors are connected to each other with DN530 Helicoflex seals and form a quasi-torus with a major radius (calculated based on the midpoints of the straight sections) of $780 \mathrm{~mm}$. Each sector consists of four cylindrical sections welded together and has two flexible bellows to reduce forces caused by thermal expansion and pressure difference. A model of the TOMAS vacuum vessel and magnetic field coils is shown in Fig. 1. An overview of the ports welded to the vacuum vessel in the vertical and horizontal planes is presented in Table I. The volume of the vessel is $\sim 1.1 \mathrm{~m}^{3}$, and its inner surface has an area of $\sim 8.5 \mathrm{~m}^{2}$.

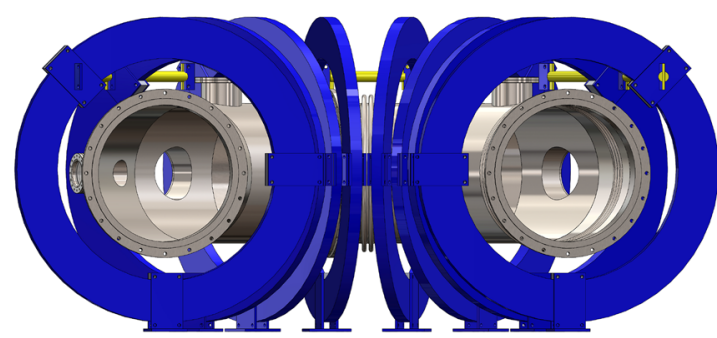

FIG. 1. The model of the TOMAS vacuum vessel and magnetic field coils.
TABLE I. An overview of the TOMAS vacuum vessel ports.

\begin{tabular}{|c|c|c|c|c|}
\hline \multirow[b]{2}{*}{ Flange size } & \multicolumn{2}{|c|}{ Vertical } & \multicolumn{2}{|c|}{ Horizontal } \\
\hline & Bottom & Top & High field side & Low field side \\
\hline DN40 & & & 2 & \\
\hline DN63 & 2 & & 2 & \\
\hline DN100 & 1 & 2 & & $4^{\mathrm{a}}$ \\
\hline DN160 & & 3 & & \\
\hline DN200 & & 1 & & 4 \\
\hline
\end{tabular}

${ }^{\mathrm{a}}$ Two out of four are tangential ports.

The vacuum vessel is equipped with 16 heating tapes allowing vacuum bakeout. Each tape provides $750 \mathrm{~W}$ of power and is attached to an individual cylindrical section with a thermocouple to monitor the local temperature of the outer wall. The maximum average temperature provided by this heating system can reach $\sim 80^{\circ} \mathrm{C}$ within approximately $45 \mathrm{~min}$. The tapes are divided into four groups of four heating tapes connected in parallel. The bakeout system is operated by using a control system (see Sec. III E), and the supplied power of each group can be adjusted separately according to the temperatures of the vacuum vessel parts.

The magnetic field is generated by 16 toroidal copper coils with an inner radius of $422 \mathrm{~mm}$ and an outer radius of $581 \mathrm{~mm}$. The centers of the coils are aligned with the centers of the corresponding vacuum vessel segments. Each coil consists of two vertical layers of copper windings with a square cross section, which has a width of $21.5 \mathrm{~mm}$. The windings are insulated from each other by a $0.3 \mathrm{~mm}$ thick insulation tape. The total amount of windings in one coil is 14. The coils are electrically connected in series and insulated from other elements of the TOMAS device. Each coil is actively cooled by water to prevent coil damage and to keep a constant resistance of $2 \mathrm{~m} \Omega$. The individual coil temperatures are monitored by using a control system. The cooling system has a primary closed water loop, which is connected via a heat exchanger to an external open loop. The heat exchanger can sustain a thermal power load up to $160 \mathrm{~kW}$. The cooling of the coils is done in parallel. The water flow and coil temperature are individually measured for each coil. Moreover, the pressure difference between the entering and leaving points of the primary loop is monitored separately.

The power supply (Munk $\mathrm{GmbH}$ ) of the magnetic field system is designed to operate coils in voltage- and current-controlled mode with the possibility to adjust the ramp-up times. The maximum output voltage and current can reach $80 \mathrm{~V}$ and $2.2 \mathrm{kA}$, respectively. The frequency of the current ripple is $300 \mathrm{~Hz}$. The accuracy of the power supply current control is $\sim 1 \%$. The value of the magnetic field is given by

$$
B(r)[T]=5.7 \times 10^{-5} \times I_{c}[A] \times \frac{R_{0}}{R_{0}+\Delta r},
$$

where $I_{c}$ is the coil current, $R_{0}$ is the major radius, and $\Delta r$ is the radial position relative to the major radius. An example of the model application in comparison to the magnetic field measurements is shown in Fig. 2.

The maximum magnetic field value of $125 \mathrm{mT}$ can be reached on the toroidal axis at the maximum current of the coils. 


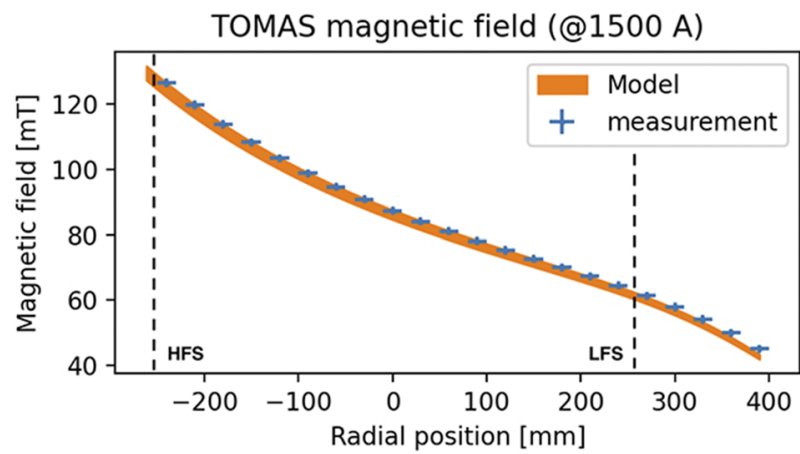

FIG. 2. A comparison of the measured and calculated magnetic field radial distributions in the equatorial plane at $I_{c}=1500 \mathrm{~A}$. The vertical dashed lines (the left one indicates the high field side border and the right one shows the low field side) allocate the space inside the vacuum vessel. The radial position of 0 corresponds to the center of the vessel. The measurements of the magnetic field are done in the plane between two coils.
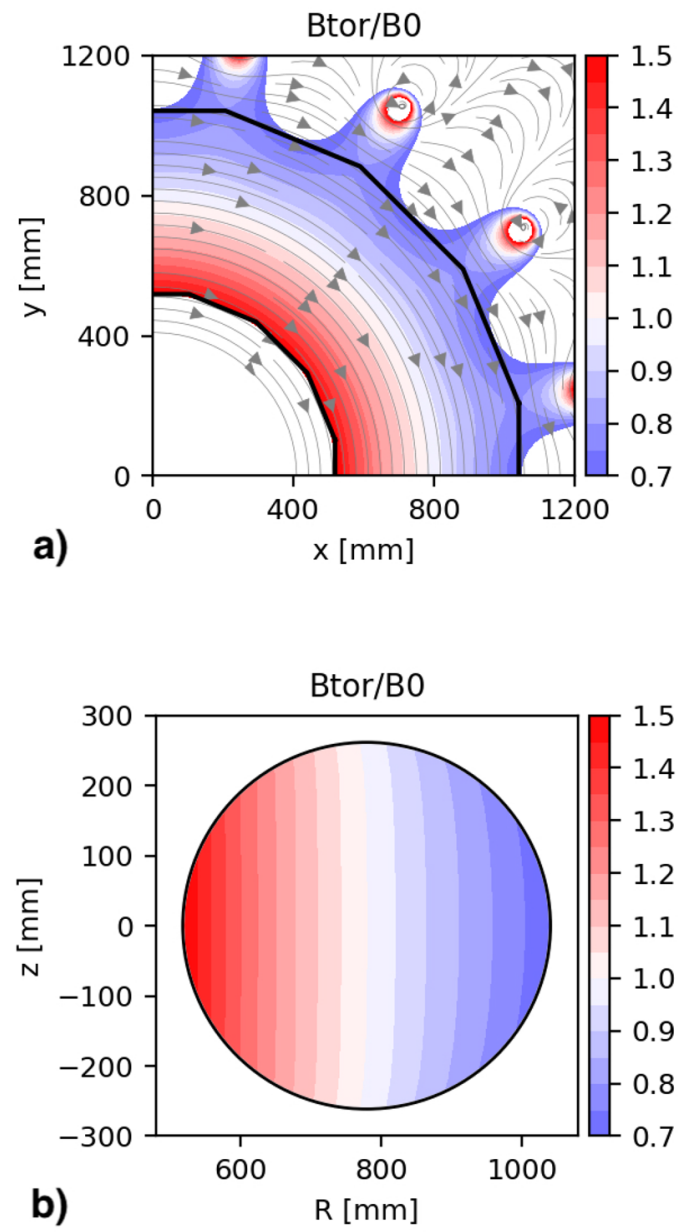

FIG. 3. The detailed model of the magnetic field intensity distribution (ratio between magnetic field values and the magnetic field value on the toroidal axis). Black solid lines indicate the area inside the vacuum vessel: (a) the toroidal cross section and (b) the poloidal cross section (in the $x-z$ plane).
The temporal magnetic field ripple is $\sim 1 \%$. The spatial ripple reaches its maximum of $6 \%$ close to the outer wall of the vacuum vessel. A detailed model of magnetic field intensity distribution computed by using Magpylib ${ }^{11}$ is shown in Fig. 3.

\section{OVERVIEW OF THE DEVICE UPGRADES}

Between 2016-2020, TOMAS went through significant refurbishment and upgrades (see Fig. 4). The device was equipped with an Ion Cyclotron Range of Frequency (ICRF) system, a new GD system, new pumping and gas injection systems, and control and data acquisition systems. The original Electron Cyclotron Resonance Heating (ECRH) system has been modified. A load-lock system with a vertical manipulator has been built on TOMAS for the exposure of material samples. Additionally, a number of new diagnostics, such as a Quadrupole Mass Spectrometer (QMS), a Time-of-Flight Neutral Particle Analyzer (ToF NPA), movable Langmuir probes, pressure gauges, and video cameras, have been installed. The first studies of wall conditioning relevant to the superconducting stellarator Wendelstein $7-\mathrm{X}^{5}$ were performed in $2017 .{ }^{12}$

\section{A. The vacuum system: Pumping, gas injection, and pressure control}

The pumping system of TOMAS consists of several pumping units. The main vacuum is provided by a Pfeiffer TPU 1201P C turbomolecular pump (turbopump), equipped by a free permanent magnetic bearing on the high vacuum side, which is fully suitable for wall conditioning, PSI studies, and other plasma physics application. The turbopump has a variable pumping speed (up to $1250 \mathrm{l} / \mathrm{s}$ for nitrogen/air) and is installed in series with a Pfeiffer DUO 35 rotary vane pump. The turbopump is connected to the vacuum vessel by a " $\Gamma$-shaped" pumping duct with a diameter of $200 \mathrm{~mm}$ and a total length of $1330 \mathrm{~mm}$. The turbopump can be isolated from the duct by an electro-pneumatic gate valve. The conductance of the pumping duct is $\sim 728 \mathrm{l} / \mathrm{s}$ (for nitrogen/air) at room temperature. Additionally, a flexible bellows connects the rotary vane pump directly to the pumping duct via a DN63 electro-pneumatic angle valve. The residual gas analysis section, the ToF NPA system, and the chamber for samples' exposures are equipped with additional pumping units. The characteristics of these systems are described in the following chapters.

The gas injection system of the device consists of two parts for which the schematic is shown in Fig. 5. The main system contains three gas inlets. These inlets are Bronkhorst F-201CV-200VGD-88-V mass flow controllers capable of injecting working gas in the range of 4 SCCM-400 SCCM (standard cubic centimeters per minute). All gas injectors are installed in parallel and operated via the control system. Each mass flow controller is coupled to a manual DN16 valve to prevent sudden leaks, to lower the background pressure in the vacuum vessel, and improve injected gas purity. The venting of the vacuum vessel is also performed via a valve installed in the lines of the main injection system. The upstream pipes of the flow controllers are connected to the gas bottles. These lines can be evacuated by using a Leybold TRIVAC D4B rotary vane vacuum pump via multiple manual valves, and their pressure is continuously monitored by using a Leybold-Heraeus Thermovac 


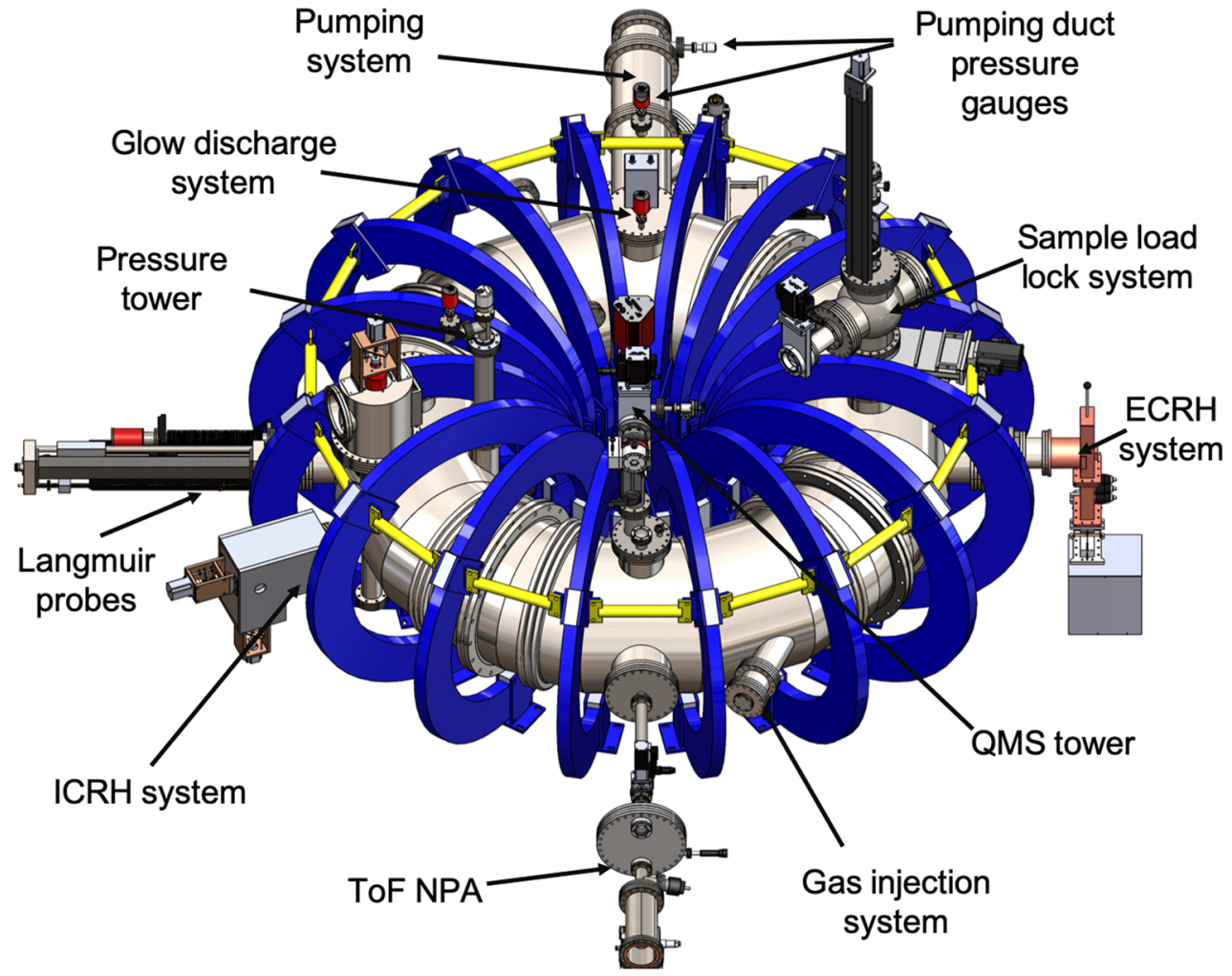

FIG. 4. The 3D model of the upgraded TOMAS device.

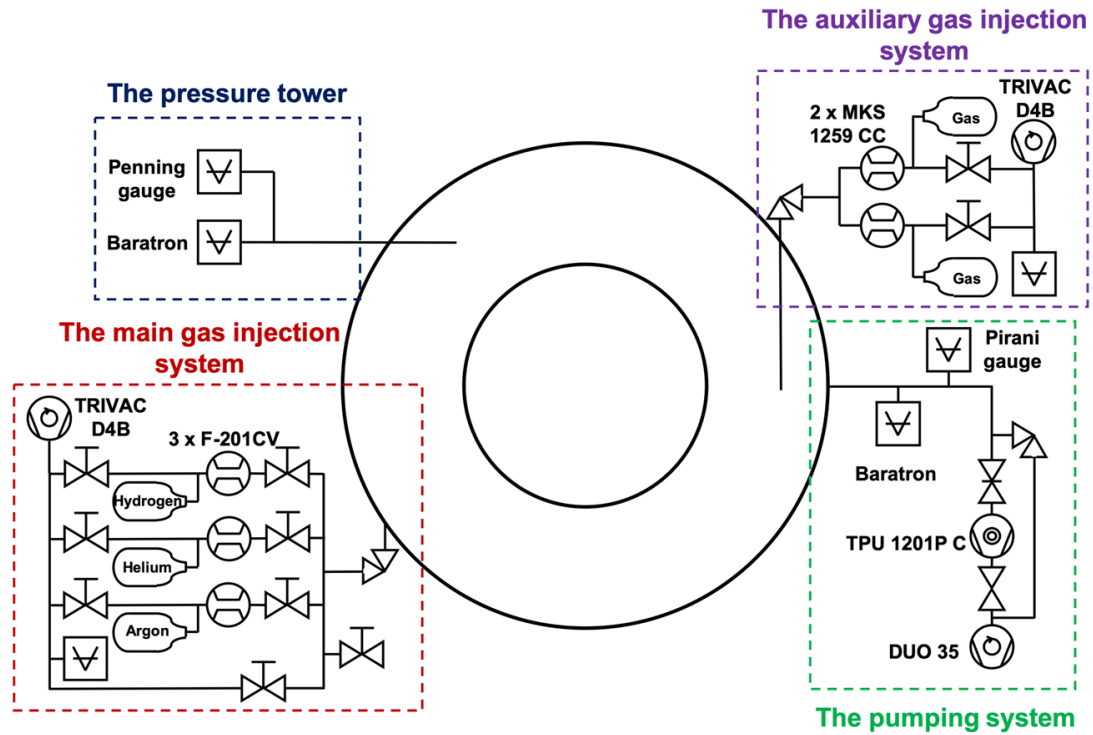

FIG. 5. The Piping and Instrumental Diagram (P\&ID) of the vacuum system of TOMAS. 
T200 pressure gauge. The connection of the gas system to the vacuum vessel is done via the DN16 flexible bellows with a length of $1000 \mathrm{~mm}$ connected via a pneumatically controlled angle valve to a reducer tee mounted on one of the horizontal tangential DN100 ports.

The second (auxiliary) gas injection system allows local gas injection in the vicinity of the glow discharge anode. It consists of two MKS Instruments 1259CC-00500RU multi-gas mass flow controllers. Each gas inlet allows us to inject up to 500 SCCM. The pumping and pressure monitoring units are of the same type as those in the main gas injection system. The gas inlets are operated by a MKS Instruments $247 \mathrm{C}$ four-channel flow controller power supply and readout.

Pressure monitoring inside the vacuum vessel is performed by several devices. The Pfeiffer CMR 375 capacitance gauge (Baratron) and the Pfeiffer IKR 270 cold cathode gauge (Penning gauge) are used for torus pressure measurements. The Baratron is used in the pressure range of $1 \times 10^{-5} \mathrm{mbar}-1.1 \times 10^{-1} \mathrm{mbar}$ and is independent of the type of gas, while the Penning gauge is reliably operated from $1 \times 10^{-9}$ mbar to $1 \times 10^{-3}$ mbar. In order to avoid any disturbance of the pressure reading by the magnetic field, both gauges are vertically installed on the top of a so-called pressure tower as it is shown in Fig. 12. The pressure tower is mounted on a vertical DN100 port close to the high magnetic field side. It connects the gauges installed on a DN40 tee via a vertical $425 \mathrm{~mm}$ long DN63 tube to the torus. The pumping duct is equipped with another Baratron and a Pfeiffer TPR 270 Pirani gauge with an operational range of $1 \times 10^{-4}$ mbar- $1 \times 10^{3}$ mbar. Optionally, a third Baratron can be installed in the vicinity of the glow anode.

Figure 6 shows the performance of the pumping and gas injection systems. An influence of the turbopump rotational speed on neutral gas pressure at the constant gas flow rates of hydrogen and helium is given in Fig. 6(a). Another example [Fig. 6(b)] presents variation of neutral hydrogen and helium pressures according to changes of corresponding gas flows at the maximum pumping speed. Both examples show a typical non-linear behavior of neutral gas pressure.

In addition, respective diagnostics also require individual pressure control units. The settings and properties of gauges used in components with differential pumping are described in Secs. III B-III E.

\section{B. Systems of plasma production \\ 1. Glow discharge system}

Glow Discharge Cleaning (GDC) is the most common wall conditioning technique applied in a large number of fusion devices. ${ }^{4}$ Studies related to GDC are also in high importance for TOMAS.

The Glow Discharge (GD) system for TOMAS has been based on that used on $\mathrm{W} 7-\mathrm{X}^{13}$ and ASDEX-Upgrade. ${ }^{14}$ It comprises a prototype W7-X glow discharge anode, which is characterized by a reliable thermal and discharge stability. ${ }^{15}$ The anode made of graphite has a spherical calotte shape with a diameter of $150 \mathrm{~mm}$ [Fig. 7(a)]. The GD anode is passively cooled mainly by radiative cooling. The maximum temperature of the anode surface would stay below $390^{\circ} \mathrm{C}$ at a maximum anode current of $3 \mathrm{~A}$ in the pressure range of $4 \times 10^{-3}$ mbar $-1 \times 10^{-2}$ mbar. ${ }^{15}$ The anode is connected to the power line via a single copper pin CF 40 vacuum feedthrough with a pin
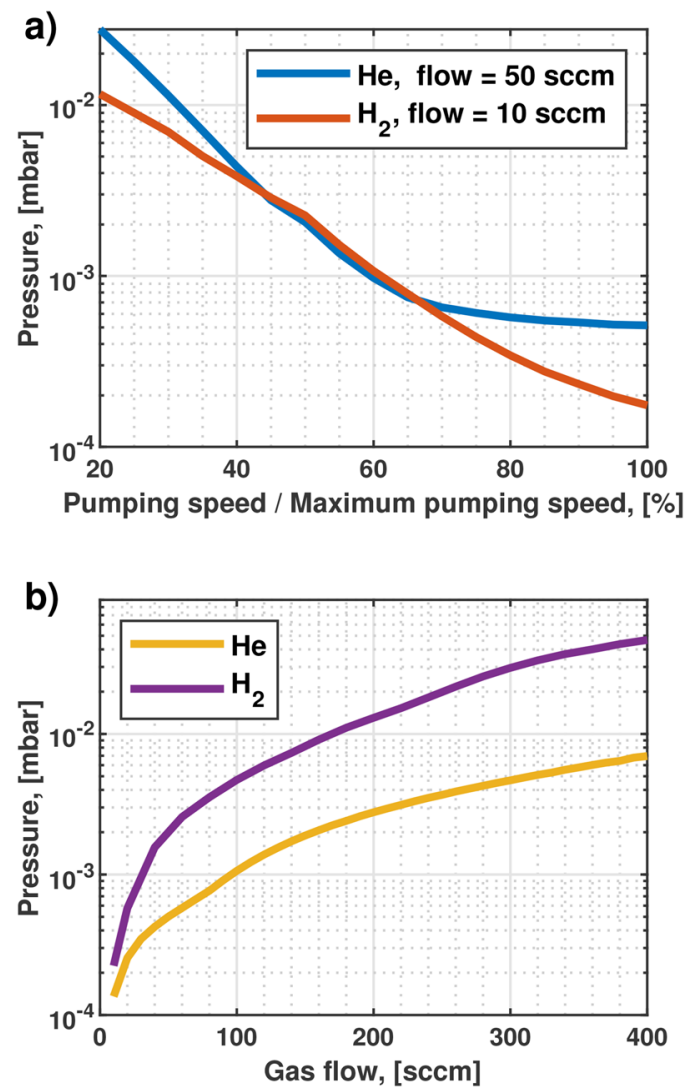

FIG. 6. Performance of the vacuum system (measured by the Baratron): (a) the pressure dependences on the pumping speed (ratio between the rotational speed and the maximum rotational speed of the turbopump) for helium at a gas flow of 50 SCCM and hydrogen at a gas flow of 10 SCCM and (b) the pressure dependences on the helium and hydrogen flows at the maximum pumping speed.

diameter of $12 \mathrm{~mm}$. The bottom of the anode spherical part [at the connection screw, see Fig. 7(b)] penetrates $\sim 10 \mathrm{~mm}$ inside the vacuum vessel. The minimum distance between the inner surface of the anode port and the edge of the anode is $25 \mathrm{~mm}$. The feedthrough is mounted on the DN200 adapter flange, which is located on the vertical port of the TOMAS device. The flange adapter is also equipped with two DN16 ports. One of these ports is used for local gas injection as described in Sec. III A. Another port is occupied by a local pressure gauge (Baratron). Thus, the GD system is suitable for studies of glow discharge breakdown assisted by local gas injections. The model of the anode and attendant components is shown in Fig. 7.

The power supply of the GD is based on the Fug MYN $60000 \mathrm{M}-1500$ modular system. The power supply can deliver a maximum voltage of $1.5 \mathrm{kV}$ and a maximum current of $40 \mathrm{~A}$. However, the current in GD operation is limited to $6 \mathrm{~A}$ to prevent any overheating and consequent damage of the power line and connection components. The power supply interlocks are integrated to the control system. The characteristic current densities of GD are $0.1 \mathrm{~A} / \mathrm{m}^{2}-0.7 \mathrm{~A} / \mathrm{m}^{2}$. The sustainable GD can be conducted in the 

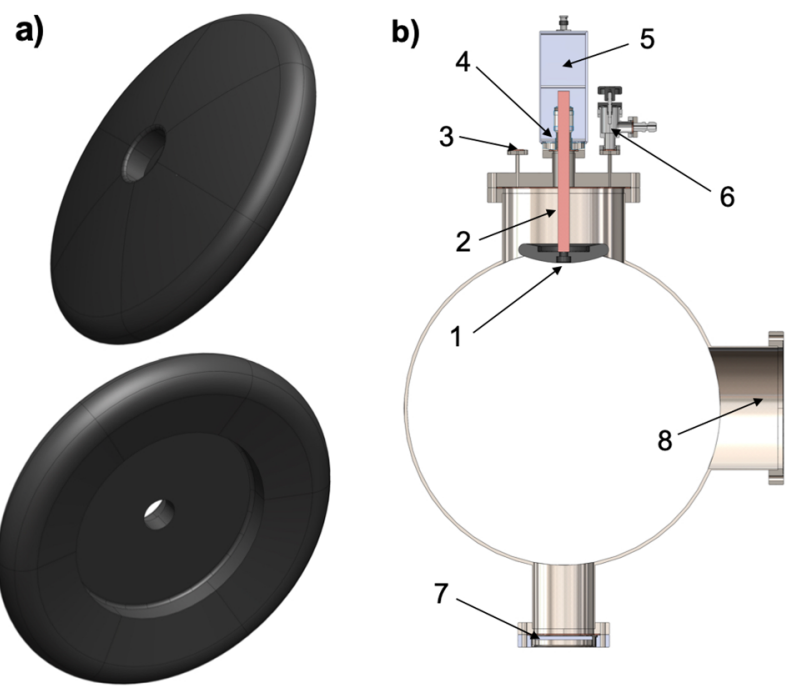

FIG. 7. (a) The model of the W7-X-like GD anode and (b) the model of the glow discharge system: (1) glow discharge anode, (2) a feedthrough connection pin, (3) DN16 port for an optional connection of the Baratron, (4) DN40 anode feedthrough, (5) electronics box, (6) DN16 manual angle valve, (7) DN100 viewport, and (8) DN200 port for the pumping system connection.

pressure range of $2 \times 10^{-4}$ mbar- $2 \times 10^{-2}$ mbar for hydrogen and $4 \times 10^{-4}$ mbar $-2 \times 10^{-2}$ mbar for helium..$^{12}$

One of the examples of glow discharge studies on TOMAS is glow discharge breakdown assisted by local gas injection. The results of the dedicated experiments are shown in Fig. 8. The glow discharge was ignited in helium (flow of 400 SCCM) with a short injection of argon (flow of up to 500 SCCM) in the vicinity of the glow anode. The voltage before breakdown was set at its maximum available value of $1500 \mathrm{~V}$, while the current was controlled at $1.5 \mathrm{~A}$ after breakdown. A small delay in the pressure response is seen in the torus compared to the glow anode port. However, the difference between the pressure measured in the anode vicinity and the pressure measured in the torus is negligible due to the limited gas flow and small dimensions of TOMAS. Nevertheless, the separate local injection allows better control of the discharge ignition process with a minor quantity of a different gas that breaks down at lower pressure. Lowering the breakdown pressure in the whole volume helps to avoid the discharge localization in the ports and vessel cavities and, thus, prevents arcing or other spurious events. The glow discharge breakdown event was assumed when the current stabilized at the set value. It was achieved within $\sim 5 \mathrm{~s}$ after the argon injection started when neutral pressure reached the level of $\sim 2.9 \times 10^{-2}$ mbar. The injection of argon was stopped right after the breakdown. The decrease in the glow discharge voltage corresponds to the decay of argon partial pressure.

\section{Electron cyclotron resonance heating system}

The original ECRH system of TOMAS ${ }^{7}$ has undergone a significant modification (Fig. 9). However, it is built around the original MUEGGE MH6000-213BF magnetron, which generates $2.45 \mathrm{GHz} 0.6 \mathrm{~kW}-6 \mathrm{~kW}$ continuous wave $(\mathrm{cW})$ output power. a)

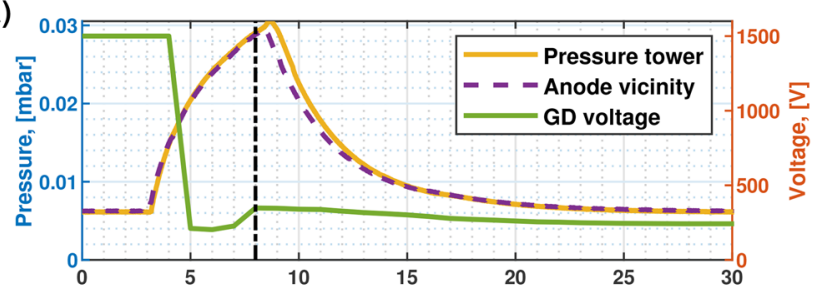

b)

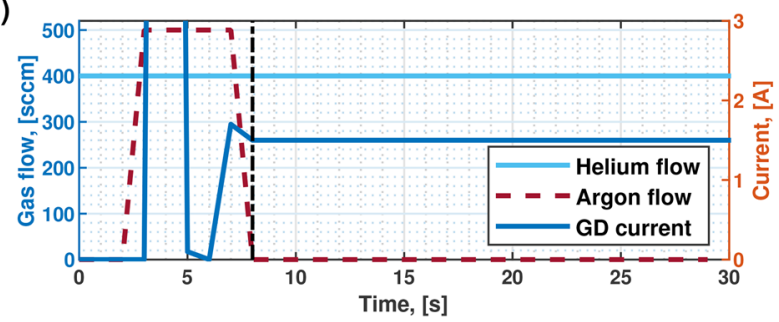

FIG. 8. The example of the glow discharge assisted breakdown experiment (black dashed line indicates the event of the breakdown). (a) Vacuum vessel neutral gas pressure, neutral gas pressure in the vicinity of the glow anode, and glow discharge voltage; (b) gas (He) flow from the main gas injection system, gas (Ar) flow from the auxiliary gas injection system, and glow discharge current.

Besides steady state operation, the MUEGGE MW-PPEE3327-6K016 magnetron power supply allows the generation of a plasma in a pulsed regime with a pulse length down to $1 \mathrm{~s}$. The pulsed regime of ECRH plasma operation is supported by an external control of the magnetron power supply by a Tektronix AFG 3252 Dual Channel Arbitrary/Function Generator. The water-cooled magnetron head is

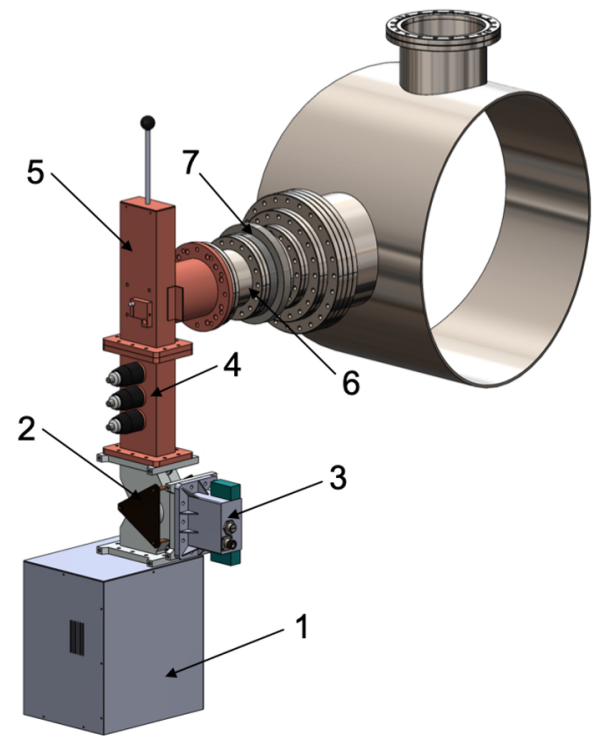

FIG. 9. The ECRH system of TOMAS. (1) MUEGGE MH6000-213BF magnetron, (2) Philips 272216302004 circulator, (3) Philips 272216302004 dummy load, (4) 3-stub tuner, (5) MUEGGE MW5002B-120YD transition element, (6) $100 \mathrm{~mm}$ DN100 pipe, and (7) fused silica UV window. 
equipped by the Philips 272216302004 isolator, which circulates the reflected power to a water-cooled power (dummy) load, to protect the system in case of an excessive load mismatch (between $2 \mathrm{~kW}$ and $6 \mathrm{~kW})$. The reflected power level is measured by a zero-bias Schottky diode (MUEGGE TD 2450-01-N microwave coaxial detector). The output flange of the magnetron is connected via a WR 340 waveguide to a $200 \mathrm{~mm}$ 3-stub tuner that contains non-contacting stubs spaced along the centerline by a quarter of the wavelength. The MUEGGE MW5002B-120YD transition element is installed after the tuner as a waveguide adapter from a vertical rectangular waveguide WR340 to a horizontal circular part with an inner diameter of $100 \mathrm{~mm}$. It is equipped with an active tuning element and an integrated adjustable short to reduce the return loss, and it maximizes the coupling of the microwave power to the load. The transition element converts the excited propagating $\mathrm{TE}_{10}$ mode in the rectangular waveguide to a $\mathrm{TE}_{11}$ mode propagating in the circular part. Following the transition element, a DN100 pipe, with a length of $100 \mathrm{~mm}$, is connected to increase the outcome of passive cooling of the transition line components. Microwave power is transmitted to the vacuum vessel through a fused silica (Synthetic Amorphous Quartz) UV window with a thickness of $6.22 \mathrm{~mm}$. The low absorption window is mounted on the DN100 viewport, which is equipped by the water-cooled frame and connected to the horizontal DN200 port of TOMAS via a $60 \mathrm{~mm}$ DN100 extension pipe and a series of standard DN adapters.

Microwaves transmitted into the vessel are partially absorbed by Electron Cyclotron Resonance (ECR). Changing the magnetic field allows us to shift a resonance layer along the diameter of the vessel in the toroidal plane; thus, different cyclotron harmonics can be achieved (Fig. 10). The visibility and the size of the resonance layer depend on the plasma discharge conditions. Partial absorption causes multiple reflections of the microwave in the vessel. It leads to multi-pass absorption and a microwave propagation and polarization mixture. ${ }^{8}$ Another dominating absorption mechanism is the upper-hybrid resonance. This resonance can be achieved where the driving magnetron frequency corresponds to the upper-hybrid resonance frequency $\omega_{u h}=\sqrt{\left(\omega_{p e}^{2}+\omega_{c e}^{2}\right)}$, where $\omega_{p e}$ is the plasma frequency, dependent on the plasma density, and $\omega_{c e}$ is the electron gyration frequency, dependent on the magnetic field. ECRH generated discharges are characterized by electron densities of

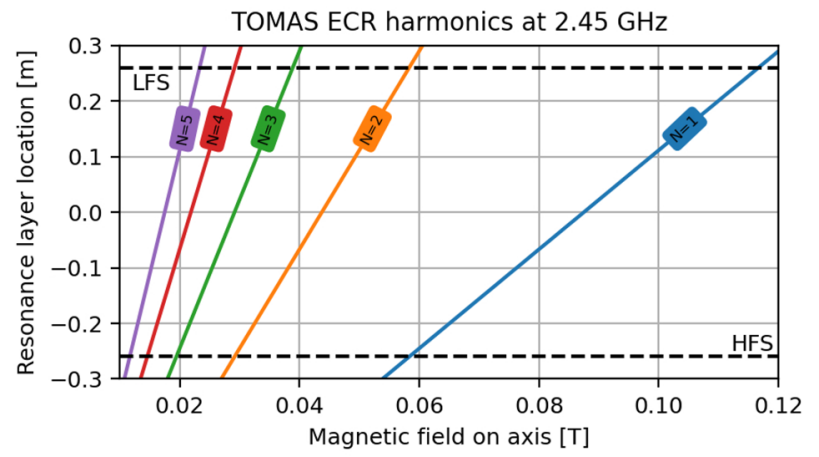

FIG. 10. Location of the cyclotron harmonic layers for the ECR as a function of the available toroidal magnetic field $\mathrm{B}_{0}$ on the toroidal axis (HFS-High field side and LFS-Low field side).
$4 \times 10^{16} \mathrm{~m}^{-3}-3 \times 10^{17} \mathrm{~m}^{-3}$ and electron temperatures of $1 \mathrm{eV}-23 \mathrm{eV}$ for hydrogen plasmas and $1.8 \times 10^{17} \mathrm{~m}^{-3}-6.6 \times 10^{17} \mathrm{~m}^{-3}$ and $8 \mathrm{eV}-15 \mathrm{eV}$ for helium plasma, respectively.

\section{Ion cyclotron range of frequency system}

The Ion Cyclotron Range of Frequency (ICRF) system was developed by the Laboratory for Plasma Physics, LPP-ERM/KMS. Based on the limited space and location of the chosen segment of the vacuum chamber and its particular geometry, an ICRF antenna was designed as a single strap antenna in an antenna box marked by lateral limiters. ${ }^{16}$

The ICRF antenna for TOMAS is shown in Fig. 11. The shape of the antenna box repeats the circular curvature of the inner wall of the vacuum vessel at the low magnetic field side. The antenna geometry was optimized for varying plasma loading conditions, which guarantees that the system can be matched in the frequency range from $10 \mathrm{MHz}$ to $50 \mathrm{MHz}$ with a power load of up to $6 \mathrm{~kW}$. The strap width is $90 \mathrm{~mm}$, while the radial distance between the antenna backside and the antenna box is $40 \mathrm{~mm}$. With a strap thickness of $5 \mathrm{~mm}$, the front face resides $5 \mathrm{~mm}$ behind the side limiters. The inner width of the antenna box is $180 \mathrm{~mm}$ with $4 \mathrm{~mm}$ thick side limiters. Both, the strap and the box, are 3D-printed in Inconel 718 and, then, coated by a thin layer of copper with a thickness of $0.2 \mathrm{~mm}-0.3 \mathrm{~mm}$ to improve the surface conductance and to relax the requirements on the complicated process of surface polishing.

Similar to the W7-X antenna matching system, ${ }^{17}$ the antenna is tuned first by using a pre-matching capacitor connected to the top of the antenna strap and fed by a center tap. The pre-matching capacitor $\mathrm{C}_{A}$ is a Comet $\mathrm{CV} 2 \mathrm{C} 1000$ variable capacitor. The central tap of the antenna is connected to a classical L-section matching circuit (Fig. 12). This circuit consists of a CVDD-1000-10S variable capacitor $\left(\mathrm{C}_{S}\right)$ connected in series and a Jennings $\mathrm{CVCH}-1000-5$ variable capacitor $\left(C_{P}\right)$ connected in parallel. The operation characteristics of the capacitors are listed in Table II. Both circuits, pre-matching and L-matching, are enclosed in the so-called pre-matching box (or top matching box) and L-matching box, respectively. The Lmatching box with dimensions of $345 \times 295 \times 190 \mathrm{~mm}^{3}$ is made of $15 \mathrm{~mm}$ thick aluminum plates. It is directly attached to the horizontal DN100 flange at the low magnetic field side. The pre-matching
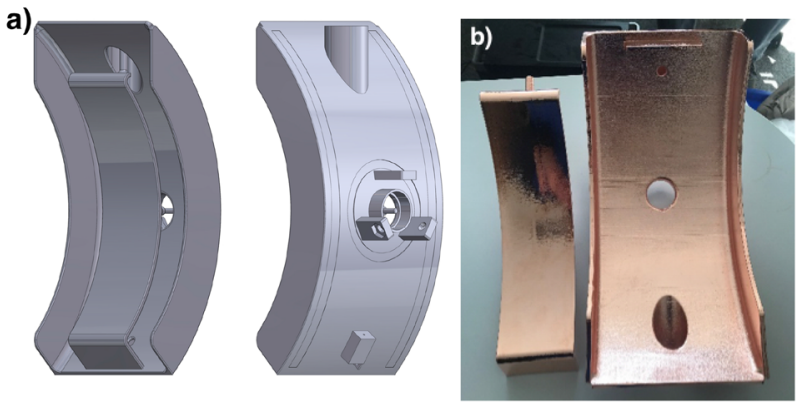

FIG. 11. The ICRF antenna for the TOMAS device: (a) the originally designed 3D model and (b) the last modified version of the antenna currently installed in the device. 


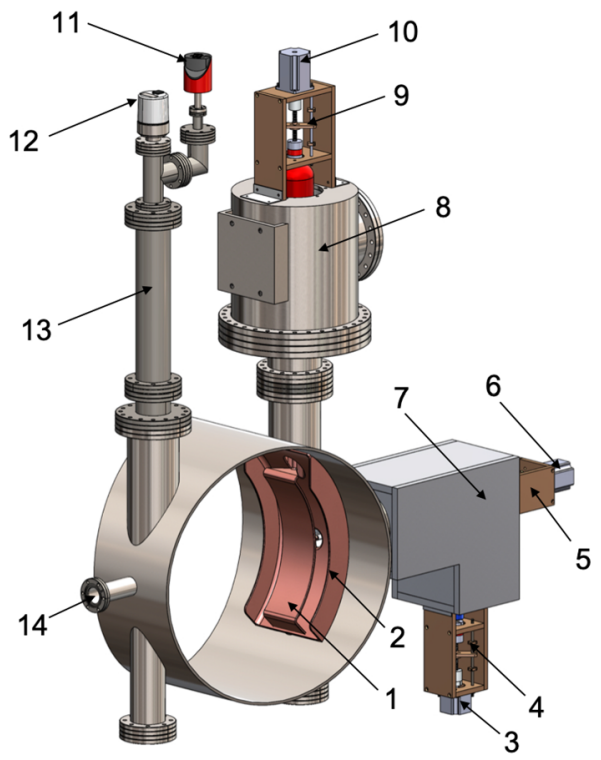

FIG. 12. The ICRF system and the pressure tower. (1) Antenna strap, (2) antenna box, (3) step motor, [(4) and (5)] automatic tuning system, (6) step motor, (7) prematching (L) box, (8) matching (top) box, (9) automatic tuning system, (10) step motor, (11) capacitance gauge Pfeiffer CMR 375 (Baratron), (12) cold cathode gauge Pfeiffer IKR 270 (Penning gauge), (13) pressure tower, and (14) camera endoscope.

box is based on a cylindrical stainless steel DN250 component of $282 \mathrm{~mm}$ height. It is mounted on top of a vertical DN100 port via a flange reducer. All vacuum capacitors are operated in an ambient pressure environment.

The matching circuits are connected to the antenna ports by $50 \Omega 15 / 8^{\prime \prime}$ rigid transmission lines going through DN40 vacuum feedthroughs that are mounted directly on the vessel ports using reducer flanges. Each capacitor is envisaged to be automatically tuned by remotely controlled step motors and several RF measurements in the matching boxes and feeding lines. The RF signals captured by voltage and current probes are read by high-speed RF logarithmic detector power meters (AD8310 0.1 MHz-440 MHz) and imported into the control system. A matching algorithm based on the TEXTOR matching scheme ${ }^{18}$ was adopted for the TOMAS ICRF system by Ref. 19 and further refined by Ref. 20. Figure 13 shows an example of the algorithm input measurements during a frequency scan around a fixed matching point. The plasma load is here a hydrogen ECRH discharge at $1.5 \mathrm{~kW}, 2.6 \times 10^{-4}$ mbar and a toroidal field

TABLE II. Matching system capacitor characteristics.

\begin{tabular}{lccc}
\hline \hline Capacitor & $\begin{array}{c}\text { Capacitance } \\
\text { range }(\mathrm{pF})\end{array}$ & $\begin{array}{c}\text { Peak voltage } \\
(\mathrm{kV})\end{array}$ & $\begin{array}{c}\text { Maximum continuous } \\
\text { operation voltage }(\mathrm{kV})\end{array}$ \\
\hline $\mathrm{C}_{A}$ & $35-1000$ & 30 & 18 \\
$\mathrm{C}_{S}$ & $25-1000$ & 10 & 6 \\
$\mathrm{C}_{P}$ & $7-1000$ & 5 & 5 \\
\hline \hline
\end{tabular}

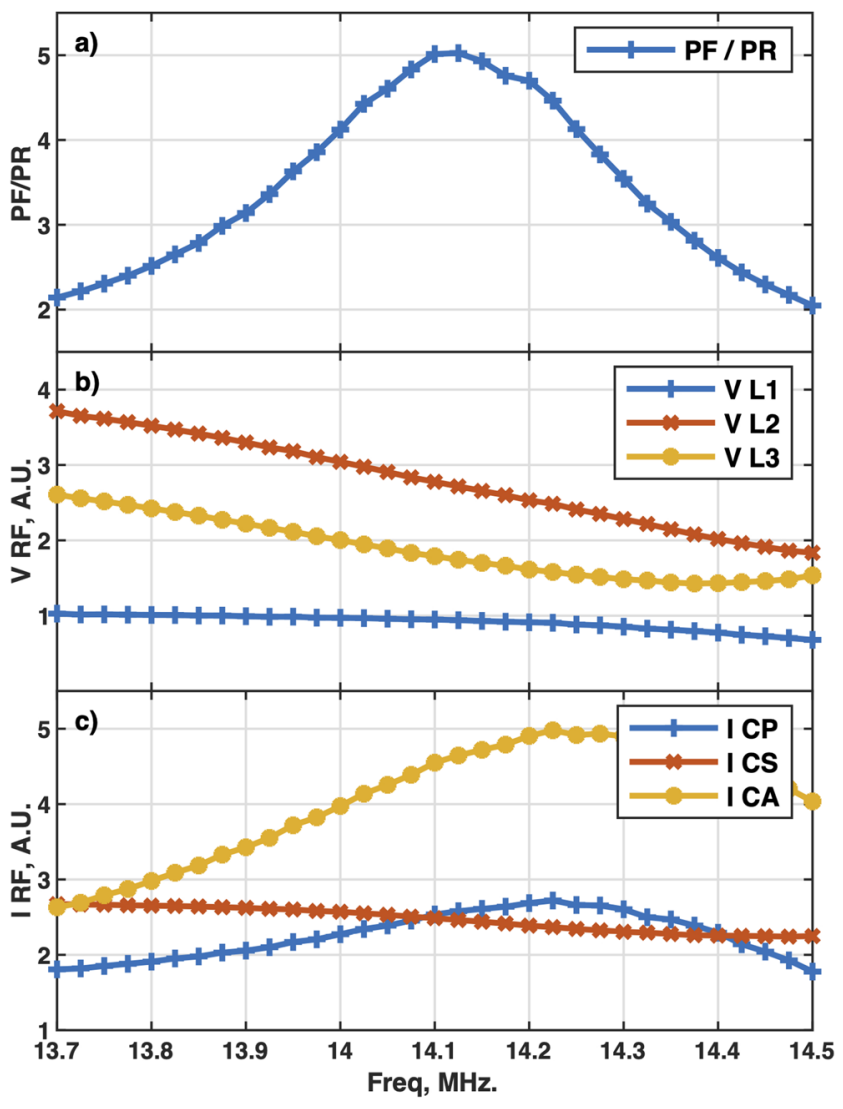

FIG. 13. Example of the input measurements that are used for the ICRF impedance matching algorithm. (a) Ratio of forward power to reflected power at the amplifier output. (b) RF voltage amplitude readings along the rigid line from the amplifier to the L-box. (c) Measurements corresponding to the RF currents though the three capacitors (see the text).

of $1400 \mathrm{~A}$. The IC power is limited to about $50 \mathrm{~W}$. Figure 13(a) shows the ratio of the forward to the reflected power, which becomes acceptable around $14.1 \pm 0.1 \mathrm{MHz}$. The algorithm (not operational during this scan) achieves impedance matching by correcting the $\mathrm{C}_{S}$ and $C_{P}$ capacitor positions according to the characteristics of the load. These load characteristics are measured by three voltage amplitude probes along the $50 \Omega 15 / 8^{\prime \prime}$ rigid transmission line between the amplifier and the L-matching box, shown in Fig. 13(b). Tuning of the pre-matching capacitor $\mathrm{C}_{A}$ is done by maximizing the currents in the top box with respect to the L-matching box; the related measurements corrected for the net IC power delivered by the amplifier are shown in Fig. 13(c).

As is the ECRH system, the ICRF system is also run by the Python controlled Tektronix AFG 3252 dual channel function generator. It allows the operation at a fixed frequency or frequency sweeps with a sweep time from $1 \mathrm{~ms}$ to $300 \mathrm{~s}$. The RF signal is amplified by using a BONN Elektronik BLWA 0105-6000P solid state amplifier working in a frequency range of $10 \mathrm{MHz}-50$ $\mathrm{MHz}$ and providing a maximum $\mathrm{cw}$ output power of $6 \mathrm{~kW}$. The amplifier RF output is monitored by using a BONN Elektronik 
BDC 0105-70/10 000 directional coupler accompanied with two low pass filters providing measurements of the forward and the reflected power in the system via the same logarithmic power meters [Fig. 13(a)].

At the low magnetic fields, appropriate for TOMAS, the main plasma operation mode is the high cyclotron harmonics $(\mathrm{HCH})$ regime with a typical frequency close to the tenth harmonics of the proton $\mathrm{H}+$. In this scheme, the electrons absorb a significant amount of the incoming power via electron-neutron collisions for both the fast wave and the Ion Bernstein wave. ${ }^{16}$ The ICRF system of TOMAS is presently operated in a pulsed regime with a discharge length of typically $4 \mathrm{~s}$. The system components are passively cooled. The dwell time between the plasma discharges, hence, depends on the plasma load and the heat dissipation in the system. The maximum RF power that can be launched is $6 \mathrm{~kW}$. The plasma density and temperature that can be achieved in ICRF discharges on TOMAS are up to $\mathrm{n}_{e}=5 \times 10^{17} \mathrm{~m}^{-3}$ and $\mathrm{T}_{e}=10 \mathrm{eV}-190 \mathrm{eV}$, respectively. The most striking difference compared to the EC discharges, and the scientific interest to install the system on TOMAS, is the presence of a significant population of energetic ions in the partially ionized plasma discharge (see Sec. III C 2), relevant for PSI, edge plasma, and RF physics studies.

\section{Diagnostics}

\section{Movable Langmuir probes}

Two types of movable Langmuir probes have been installed into a horizontal DN200 port [Fig. 14(a)] to provide measurements of the main plasma parameters, such as the electron density and temperature, the plasma and floating potentials, and their radial distributions along the diameter of the vacuum vessel. Both probes are operated at the toroidal mid-plane (equatorial plane) [Fig. 14(b)]. The distance between the probes' axes is $138 \mathrm{~mm}$ at the low magnetic field side. The reason to use two different probes at almost the same location is to provide reliable measurements of plasma parameters applying cross-calibration and complementary measurements.

One of the probes is a commercial single Langmuir Probe manufactured by Impedans Ltd. The system consists of the Langmuir probe within a manipulator equipped by an automated linear drive and a data acquisition unit. The system is connected to the vacuum vessel via a flexible bellows with DN40 flanges. The current probe configuration uses a custom-made single tip probe head (Fig. 14). The probe head has a cylindrical shape with a diameter of $10 \mathrm{~mm}$ as well as a probe shaft, which embodies the Langmuir probe. Both components have a ceramic cover that holds the probe tip and isolates the probe cradle. The total length of the probe is $400 \mathrm{~mm}$. The tip is made of tungsten and has a shape of the right circular cylinder with a height of $1.4 \mathrm{~mm}$ surmounted by the hemisphere with a diameter of $1.5 \mathrm{~mm}$. The probe has parameter ranges of the floating potential between $-145 \mathrm{~V}$ and $145 \mathrm{~V}$, the plasma potential is between $-100 \mathrm{~V}$ and $145 \mathrm{~V}$, the collected current range is between $-150 \mathrm{~mA}$ and $150 \mathrm{~mA}$ with the resolution down to $1 \mu \mathrm{A}$, plasma densities range from $1 \times 10^{11} \mathrm{~m}^{-3}$ to $1 \times 10^{18} \mathrm{~m}^{-3}$, the electron temperature is between $1 \mathrm{eV}$ and $20 \mathrm{eV}$, and the electron energy probability function can have values from $0 \mathrm{eV}$ to $100 \mathrm{eV}$. The range of the probe voltage scan is $-150 \mathrm{~V}$ to $150 \mathrm{~V}$. Additionally, the single Langmuir probe uses RF and DC compensation schemes to minimize the RF voltage drop across the probe sheath and to measure changes in the plasma floating potential, respectively.

As an example of the single Langmuir probe operation, the radial distribution of the electron density and temperature for hydrogen ECRH plasmas is given in Fig. 15(a). In this case, the measurements were done only for the low magnetic field side part of the vessel due to the limited size of the probe manipulator shaft. This radial profile scan shows a density maximum toward the low field side limited by the radial extend of the ICRF antenna. In addition, the maximum temperature is located at the low magnetic field side
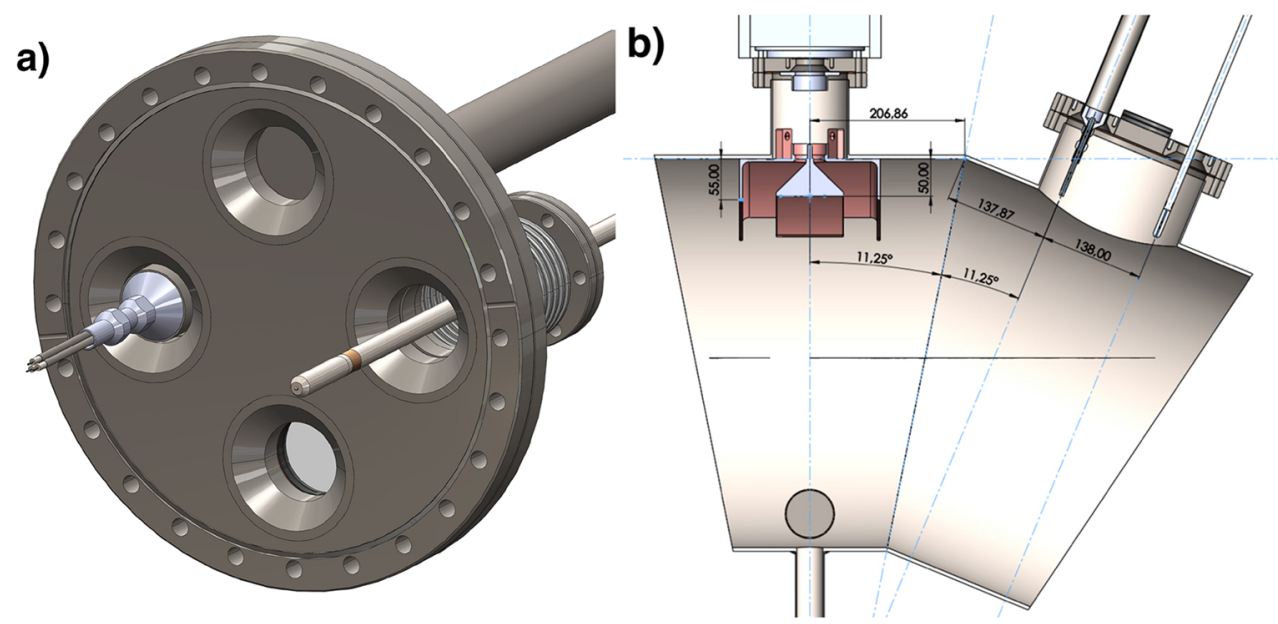

FIG. 14. The 3D model of the Langmuir probes. (a) The probe installation in the horizontal DN200 port. (b) Probe locations and dimensions relative to the ICRF antenna in the equatorial plane. 

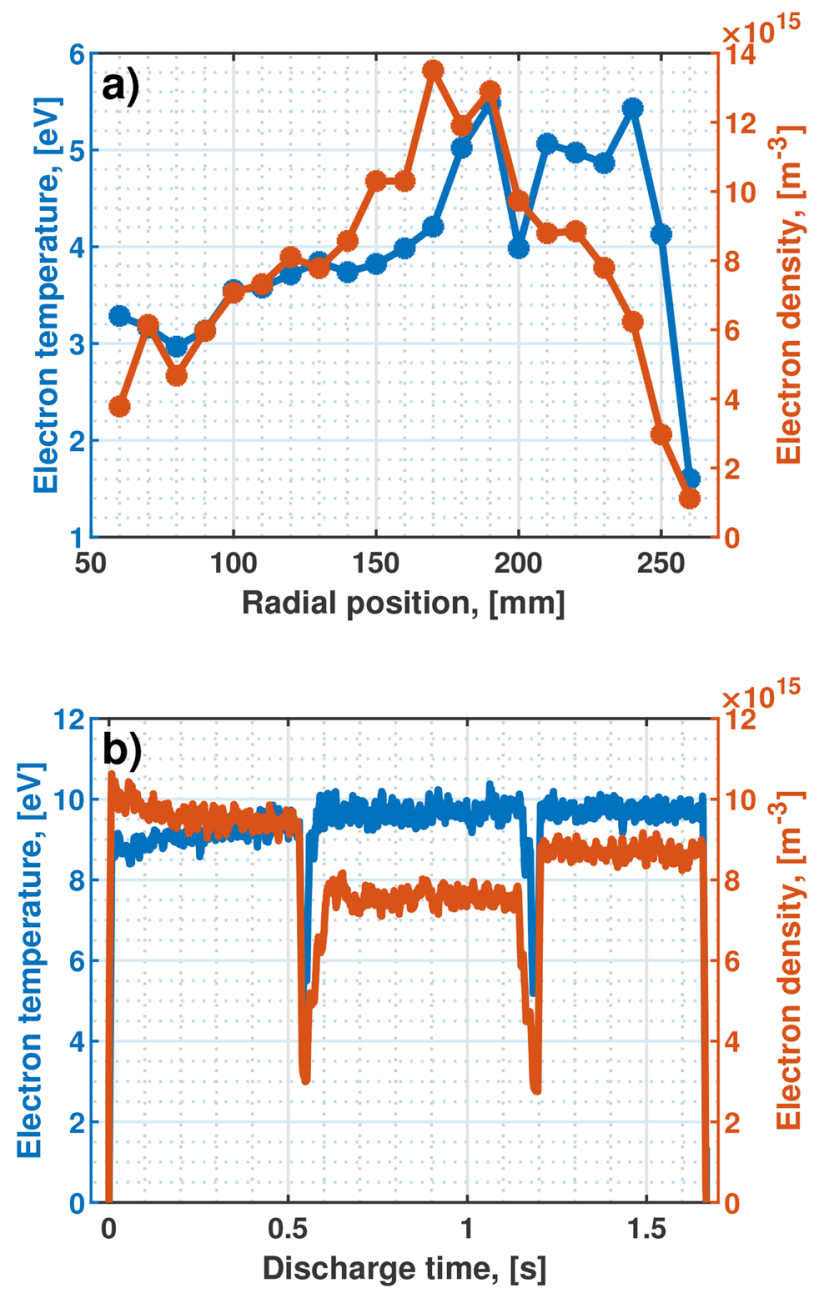

FIG. 15. (a) The example of the single Langmuir probe operation: radial distribution of electron density and temperature in the hydrogen ECRH discharge (ECRH power is $815 \mathrm{~W}$, magnetic coil current of $1350 \mathrm{~A}$, neutral gas pressure $\sim 2 \times 10^{-4}$ mbar, and discharge length is $2 \mathrm{~s}$ ). (b) The example of the triple Langmuir probe operation: Time evolution of electron temperature $T_{e}$ (red line) and electron density $\mathrm{n}_{e}$ (blue line) in RF discharge (hydrogen flow is $35 \mathrm{SCCM}$, radial probe position is $152 \mathrm{~mm}$ relative to the toroidal axis, and $\mathrm{B}_{0}=0.114 \mathrm{~T}$ ).

in this plasma as a result of the typical transport in a simple toroidal magnetic field configuration.

The second movable Langmuir probe is a triple probe. This probe type, proposed in Ref. 21, can be used to diagnose plasmas driven with and without a magnetic field. The operation of the triple probe does not require a voltage or frequency sweep in comparison to other Langmuir probe methods ${ }^{22}$ and allows determining instantaneous values of plasma parameters.

A triple probe head [Fig. 14(a)] was designed and manufactured by the Institute of Plasma Physics, NSC KIPT. It consists of a stainless steel holder that embodies three tips insulated by cylindrical ceramic covers enclosed in stainless steel thin pipes, which seal and protect the ceramics from mechanical stress. The inner diameter of the probe holder is $9 \mathrm{~mm}$. The diameter of the ceramic cover is $2.6 \mathrm{~mm}$, while the outer diameter of the pipes is $4 \mathrm{~mm}$. The triple probe tips are made of a tungsten cylindrical wire. The length of the tip is $4 \mathrm{~mm}$, while its diameter is $0.8 \mathrm{~mm}$. The tips are located equidistantly with a separation of $3.5 \mathrm{~mm}$ between each pair of tips. The triple probe head is installed inside the $34 \mathrm{~mm}$ diameter shaft of an automated linear-drive manipulator. The tips of the probe are connected to a measuring electrical circuit similar to Ref. 21. Circuit outputs are connected via coaxial cables to the data acquisition system. The measurement limitation of the parameter range is defined mostly by the data acquisition system.

Additionally, an identical movable triple probe system is available for the top vertical DN160 port of the same vacuum vessel segment to perform a vertical scan of plasma parameters.

As an illustration of the triple probe operation, the temporal evolution of plasma parameters for an ICRF discharge is shown in Fig. 15(b). The discharge has the following parameters: The generator frequency is $24.9 \mathrm{MHz}$. The RF power is variated stepwise. The duration of each step is $0.5 \mathrm{~s}$. The maximum power of $\sim 1 \mathrm{~kW}$ is achieved at the first step. The second and the third discharge steps have $\sim 80 \%$ and $\sim 90 \%$ of the maximum value of the RF power, respectively. The power between steps is reduced to a minimum value, which is $\sim 30 \%$ of the maximum power level. As one can see in Fig. 15(b), the electron density changes proportional to the power, while the electron temperature slightly increases regardless the power variation from step to step. Between discharge steps, the electron density and temperature decrease by $\sim 3$ times and by $\sim 2$ times, respectively. Such dependence of the plasma parameters is typical for low-temperature weakly ionized plasma. This example shows the suitability of the triple probe to study variations of the RF discharge parameters.

Both probes have also been tested together in hydrogen and helium plasma ECRH discharges. The results of these experiments show a satisfactory agreement between plasma parameters obtained by both probes. ${ }^{23}$ Thus, for example, the difference of the electron temperature measurements is not greater than $25 \%$.

\section{Time-of-flight neutral particle analyzer}

A Neutral Particle Analyzer (NPA) was installed on TOMAS to determine neutral fluxes, which are of particular interest for RF plasma applications. Based on the Time-of-Flight (ToF) principle, this type of diagnostics allows us to see and study the absorption of RF power by ions via the energy spectra of the charge exchange neutrals. The diagnostic is well suited for plasma studies on TOMAS. It was previously operated on the reversed field pinch fusion device EXTRAP-T2R ${ }^{24}$ A detailed description of the working of the ToF NPA and its mechanical structure is given in Ref. 25. The original equipment underwent a few modifications during its installation and integration on TOMAS. The model of the diagnostic as it is mounted in one of the TOMAS section can be seen in Fig. 16.

The ToF NPA consists of the following parts, starting from the vacuum vessel, a section between the vacuum vessel and a chopper, a chopper tower, a flight path, and a detector tower. The first section connects the diagnostic to the vacuum vessel via a horizontal DN200 port. The DN40 adapter flange is extended by the $500 \mathrm{~mm}$ DN40 pipe to place the DN40 electro-pneumatic gate valve outside the area between the magnetic field coils. The valve separates the volumes of 


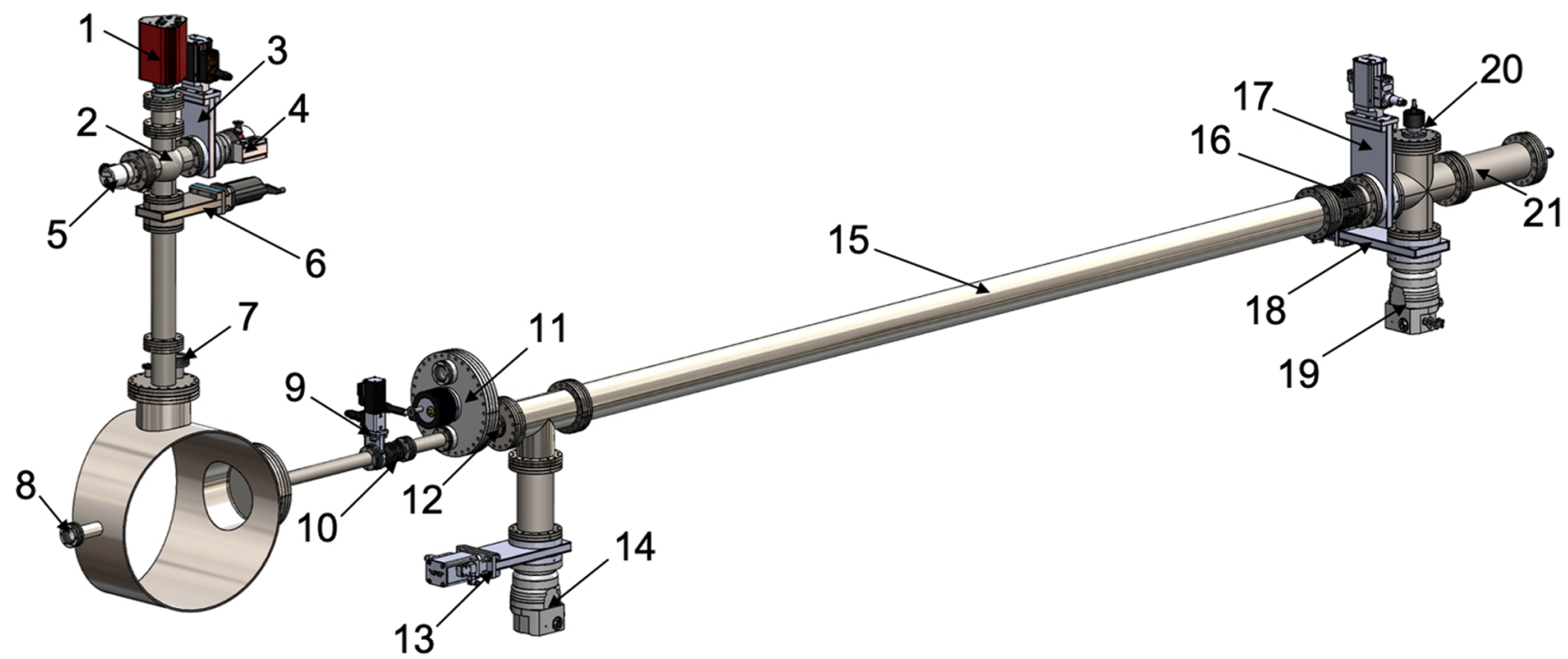

FIG. 16. Time-of-Flight neutral particle analyzer and residual gas analyzer. (1) Quadrupole Mass-Spectrometer (QMS), (2) by-pass DN16 angle valve, (3) DN63 electropneumatic gate valve, (4) Pfeiffer HiPace 80 turbomolecular pump, (5) Pfeiffer IKR 270 cold cathode gauge (Penning gauge), (6) DN63 manual gate valve, (7) DN40 viewport, (8) DN40 viewport, (9) DN40 electro-pneumatic gate valve, (10) DN40 bellows, (11) chopper, (12) Leybold PENNINGVAC PR 27 pressure gauge, (13) DN100 electro-pneumatic gate valve, (14) Pfeiffer HiPace $300 \mathrm{H}$ turbomolecular pump, (15) DN100 flight distance pipe, (16) DN100 bellows, (17) DN100 electro-pneumatic gate valve, (18) DN100 electro-pneumatic gate valve, (19) Pfeiffer HiPace $300 \mathrm{H}$ turbomolecular pump, (20) Leybold PENNINGVAC PR 27 pressure gauge, and (21) detector pipe.

the vacuum vessel and the diagnostic. A bellows after the valve provides mechanical insulation between the vessel port and the chopper tower.

The chopper consists of a small cylindrical vacuum chamber with a width of $50 \mathrm{~mm}$ based on the DN250 vacuum flange elements. Each side of the chamber is equipped by two DN40 ports symmetrically placed in reference to the chamber center. There are two $3 \mathrm{~mm}$ thick aluminum coaxial disks enclosed inside the chopper chamber. One of the disks with a diameter of $250 \mathrm{~mm}$ is fixed and has two radial $0.15 \times 40 \mathrm{~mm}^{2}$ rectangular slits $180^{\circ}$ apart. Another disk is a rotating disk with a diameter of $240 \mathrm{~mm}$. It is connected to the Elettrorava M52-25 motor allowing to achieve a rotation speed of up to $20000 \mathrm{rpm}$. The rotating disk has 20 radial slits with the same size as the fixed disk. The slits have an angular separation of $18^{\circ}$. The slits of both disks are centered on the axis of the DN40 port. The port is used for the connection of the chopper chamber to the other part of ToF NPA. A linear-drive feedthrough attached to the side of the chopper chamber is used to change the aperture size of the chopper by moving a connected V-shaped metal plate along the disk surface perpendicular to the axis of one of the ports. Another pair of DN40 ports is equipped by borosilicate glass viewports and used for triggering of the NPA detector. A trigger signal is generated by combination of a $5 \mathrm{~mW} \mathrm{He}-\mathrm{Ne}$ laser (LGK7621) and a photo transistor, which are placed on each side of the viewports and shaped by a small operation amplifier differentiating circuit to avoid effects of changes in light levels. The timing of this signal is then recorded with the CAEN DT5790 digital acquisition system (see below).
Behind the chopper, a DN40 tee is mounted on which the pressure gauge (Leybold PENNINGVAC PR 27) is installed to monitor the pressure in the section of the chopper tower and the flight distance tube. It is followed by the DN100 tee, which is connected through an adapter flange. That piece accommodates an electropneumatic gate valve, which gives access to the chopper tower pumping unit based on the Pfeiffer HiPace $300 \mathrm{H}$ turbopump (with a maximum pumping speed of $260 \mathrm{l} / \mathrm{s}$ ). The pumping unit provides differential pumping of the chopper tower and the flight distance pipe. The chopper tower has its own support structure, which places the central axis of the tower at the same level as the equatorial plane of the vacuum vessel.

Following the chopper tower, there is the DN100 flight distance pipe with a length of $3000 \mathrm{~mm}$. Inside the pipe, there is an equidistant set of $1 \mathrm{~mm}$ thick copper collimator plates with a central circular aperture of $35 \mathrm{~mm}$ diameter. The disks are fixed in place by a system of rods. This installation is aimed at preventing the arrival of stray radiation and particles to the detector. The opposite end of the pipe is connected to a bellows that provides a mechanical insulation between the flight distance pipe and the detector tower.

The detector tower follows at the end of the flight distance pipe. The tower is mounted on a special support platform, which is designed to provide good electrical insulation, alignment capabilities, and mechanical stability. The central component of the detector tower is a 4-way DN100 cross. One of the horizontal ports of the cross is separated from the flight distance tube by an electro-pneumatic valve, allowing the vacuum volume of the detector tower to be differentially pumped. The bottom part of the cross 
is connected through another electro-pneumatic valve to the Pfeiffer HiPace $300 \mathrm{H}$ turbopump, which allows us to keep the detector at low pressure independently. The pressure of the section is monitored by using the pressure gauge (Leybold PENNINGVAC PR 27) mounted on the top of the 4-way cross. The opposite horizontal port of the cross is connected to a $335 \mathrm{~mm}$ DN100 pipe, which contains the detector inside. The pipe is electrically insulated from the rest of the diagnostic by using a Vespel gasket.

The detector used in ToF NPA is the Hamamatsu R595 head-on electron multiplier. The electron multiplier tube has 20 box-andgrid-type stages separated by a $1 \mathrm{M} \Omega$ resistor. Each stage consists of a quarter cylindrical $\mathrm{Cu}-\mathrm{BeO}$ dynode. The active area of the sensor is $10 \times 12 \mathrm{~mm}^{2}$. The detector is mounted inside a double magnetic shield. The outer magnetic shield is fixed to a DN100 flange, thus assuring that the detector window is on the ToF NPA axis. The assembly of the support and the magnetic shields is done using a DN100 flange, which is also equipped with a 10-pin electrical feedthrough for the powering of the detector and the signal output. The experimental results are a set of numeric data of timing and gate signal, and they are recorded by using the CAEN DT5790 dual digital acquisition system. The acquisition system is based on a $2 \times 250 \mathrm{MS} / \mathrm{s}$ and 12-bit waveform digitizer. It is also combined with a power supply containing two $\pm 12 \mathrm{~V} 100 \mathrm{~mA}$ and $\pm 24 \mathrm{~V} 50 \mathrm{~mA}$ low voltage bias outputs and two $\pm 4 \mathrm{kV} 3 \mathrm{~mA}$ ( $4 \mathrm{~W}$ max.) high voltage bias outputs.

In the final layout, the diagnostic is aligned in the radial direction looking toward the center of TOMAS. The distance between the slits of the chopper and the first dynode of the detector is $3820 \mathrm{~mm}$, and the solid angle under which the detector is seen from the slits is $8.2 \mu \mathrm{Sr}$. The neutral particle energy range of the system is $10 \mathrm{eV}-1000 \mathrm{eV}$. Here, the lower limit is defined by the detector sensitivity, while the upper limit is determined by the time resolution. At the full rotational speed, the interval for consecutive passages of the rotating slits in front of the stationary slit is about $150 \mu \mathrm{s}$, and this, consequently, provides the slit opening time about $1.8 \mu$ s for particle pass through.

The data registered by the NPA in the selected time range are integrated for pulses performed under the same experimental conditions. The integrated distribution of neutral particles reflects the particle energy distribution in the plasma. Figure 17 shows one example for the photons and neutral particles reaching the detector forming an arrival time distribution (ATD). The example is integrated over 18 pulses and normalized by the total plasma time under the same conditions in a series with the hydrogen flow of 35 SCCM. The blue curve corresponds to the $2 \mathrm{~s} 2 \mathrm{~kW}$ EC and $1 \mathrm{~kW}$ IC plasma discharges. The orange curve shows the results for $2 \mathrm{~s} 1 \mathrm{~kW} \mathrm{IC}$ plasma discharges. The ATD corresponds to the inverted velocity distribution of neutrals from the plasma.

\section{Residual gas analyzer}

A key diagnostic in the wall conditioning and plasma surface interaction studies on TOMAS is the Residual Gas Analyzer (RGA). The main element of this diagnostic is a Quadrupole Mass Spectrometer (QMS) Pfeiffer PrismaPlus QMG 220 M2. The type of the QMS detector is a Faraday cup. The QMS has a grid ion source that is equipped with two tungsten filaments. The QMS system is run via Quadera ${ }^{\circledR}$ software. It can operate within the mass range of

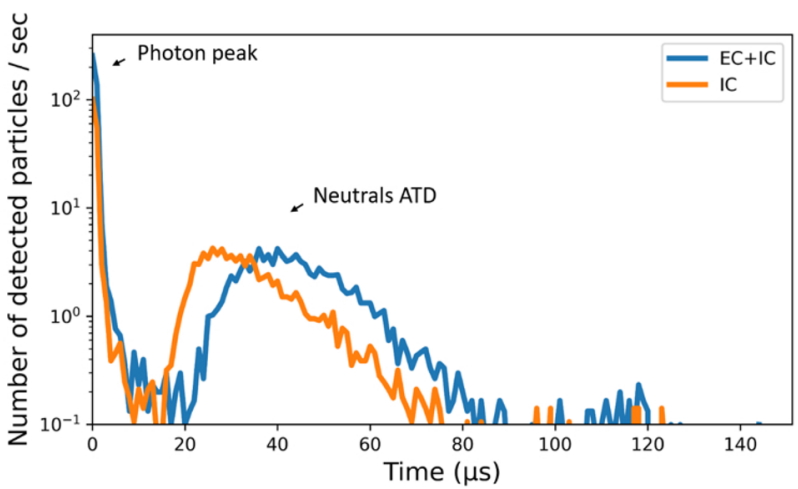

FIG. 17. Integrated and normalized NPA results in TOMAS comparing only $1 \mathrm{~kW}$ $\mathrm{IC}$ plasma with combined $1 \mathrm{~kW} \mathrm{IC}$ and $2 \mathrm{~kW}$ EC plasmas.

$1 \mathrm{u}-200 \mathrm{u}$. The system has a resolution down to 0.5 . The scan speed of the QMS is from $100 \mathrm{~ms} / \mathrm{u}$ to $1 \mathrm{~s} / \mathrm{u}$ in Multi-Ion Detection (MID) mode and from $100 \mathrm{~ms} / \mathrm{u}$ to $1 \mathrm{~s} / \mathrm{u}$ in analog scan mode. The operating pressure range of the QMS is $1 \times 10^{-8}$ mbar- $1 \times 10^{-5}$ mbar. Additionally, the system is also used to perform a leak detection test of the vacuum system.

QMS is vertically installed on the top of the so-called RGA tower (Fig. 16). This construction is built to prevent any direct contact of plasmas with the QMS active elements and to minimize the influence of the magnetic field on the spectrometer performance. The tower consists of DN63 vacuum parts. An enlarged 4-way DN63 cross serves as the base for the QMS, pumping, and pressure control elements. The cross is elevated $430 \mathrm{~mm}$ ( $360 \mathrm{~mm}$ without a gate valve) above the vacuum vessel top port. It is separated from the main volume of the vacuum system by using a manual DN63 gate valve, which allows us to control particle flows precisely according to neutral pressure in the vacuum vessel. The RGA tower is installed on the DN160 port via a flange reducer, which also contains DN40 and DN16 flanges used for a viewport installation and a vacuum by-pass connection, respectively. The by-pass to the RGA vacuum section, connected via an additional DN16 side flange on the 4-way cross, allows us to control the conductance of the neutral gas to very low levels allowing the reliable work of the diagnostic at pressures of up to $6 \times 10^{-2} \mathrm{mbar}$ in the main vacuum vessel.

The RGA section has a differential pumping, which is controlled independently from the main vacuum system. The section volume can be pumped down to $2 \times 10^{-8} \mathrm{mbar}$ by the Pfeiffer HiPace 80 turbomolecular pump, providing the maximum pumping speed of $67 \mathrm{l} / \mathrm{s}$ (for nitrogen/air) for the RGA section volume of $1.53 \mathrm{l}$. The section pressure is monitored by using the Pfeiffer IKR 270 cold cathode gauge (Penning gauge). Both parts are installed in the horizontal ports of the 4-way enlarged DN63 cross.

An example of QMS operation is demonstrated for an experiment dedicated to the vacuum vessel baking (Fig. 18). Baking is the first wall conditioning technique, which is used in the initial conditioning phase to prepare the device for the operation after the device pump down. The bakeout was performed for $65 \mathrm{~min}$. The whole procedure consisted of the temperature rise phase and the temperature plateau phase. The maximum average temperature of the vessel wall 

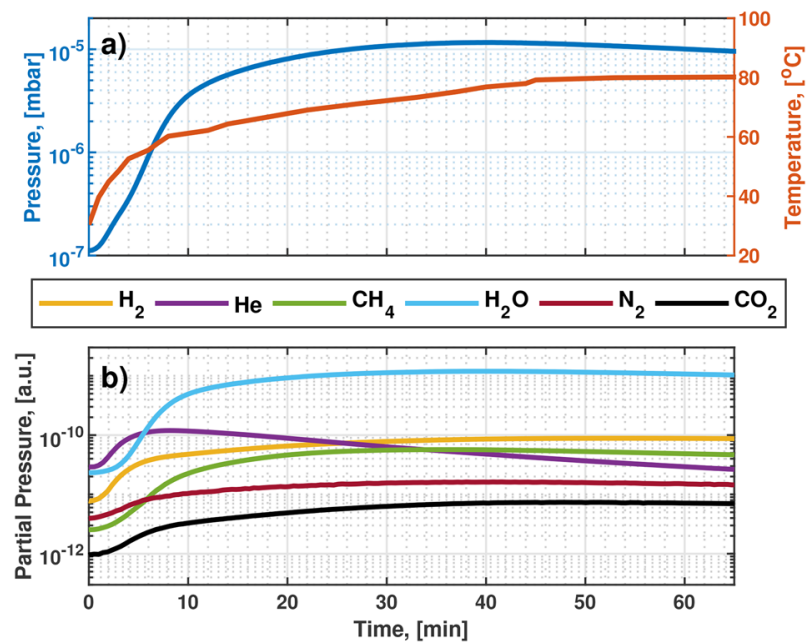

FIG. 18. Results of the TOMAS vacuum vessel baking. (a) Time evolution of average temperature of the vacuum vessel walls and neutral gas pressure (base pressure) and (b) time evolution of the residual gas content obtained from QMS measurements.

was $80^{\circ} \mathrm{C}$ (the plateau phase) and was achieved after $\sim 45 \mathrm{~min}$ of operation [Fig. 18(a)]. The total residual gas pressure raised up to $\sim 1.1 \times 10^{-5}$ mbar in the first phase due to the temperature stimulated outgassing from the vessel walls. The pressure maximum occurred about $5 \mathrm{~min}$ before the average temperature achieved its maximum, thus demonstrating the effective desaturation of the surfaces by baking. Figure 18(b) shows the behavior of the impurities dominating in the residual gas content obtained by the QMS. The spectrum shows that water $\left(\mathrm{H}_{2} \mathrm{O}\right)$ is the most prominent species. The water signal repeats the shape of the total residual gas pressure as well as methane $\left(\mathrm{CH}_{4}\right)$ and nitrogen $\left(\mathrm{N}_{2}\right)$. The concentration of released helium $(\mathrm{He})$ remaining from previous plasma operations achieved its highest point after $6 \mathrm{~min}-7 \mathrm{~min}$ of baking, which was followed by a strong decrease pattern. On contrary, hydrogen and carbon dioxide $\left(\mathrm{CO}_{2}\right)$ outgassing reached its maxima only in the plateau phase. This type of impurity outgassing during the baking is typical for devices with a full stainless steel wall and small graphite elements (glow anode).

\section{Video diagnostics}

To monitor and record a wide variety of plasma events in the TOMAS device, several video diagnostics have been installed. Three Logitech C615 USB HD video cameras are used to observe the plasma from different positions, as it is shown in Fig. 19(a). The cameras have an image resolution of up to $8 \mathrm{MP}$. The maximum video resolution of this type of cameras is $1920 \times 1080$ pixels with a frame rate of $30 \mathrm{fps}$. Each camera is equipped by a glass element focal system with an autofocus function. The Field of View (FoV) is $78^{\circ}$. The cameras are mounted on viewports with borosilicate glass (Kodial). Each viewport is protected by a metal mesh (size of wire is $0.05 \mathrm{~mm}$, cell size is $1 \times 1 \mathrm{~mm}^{2}$ ) attached to the vacuum side of the viewports to protect the workspace against microwave radiation leaks.
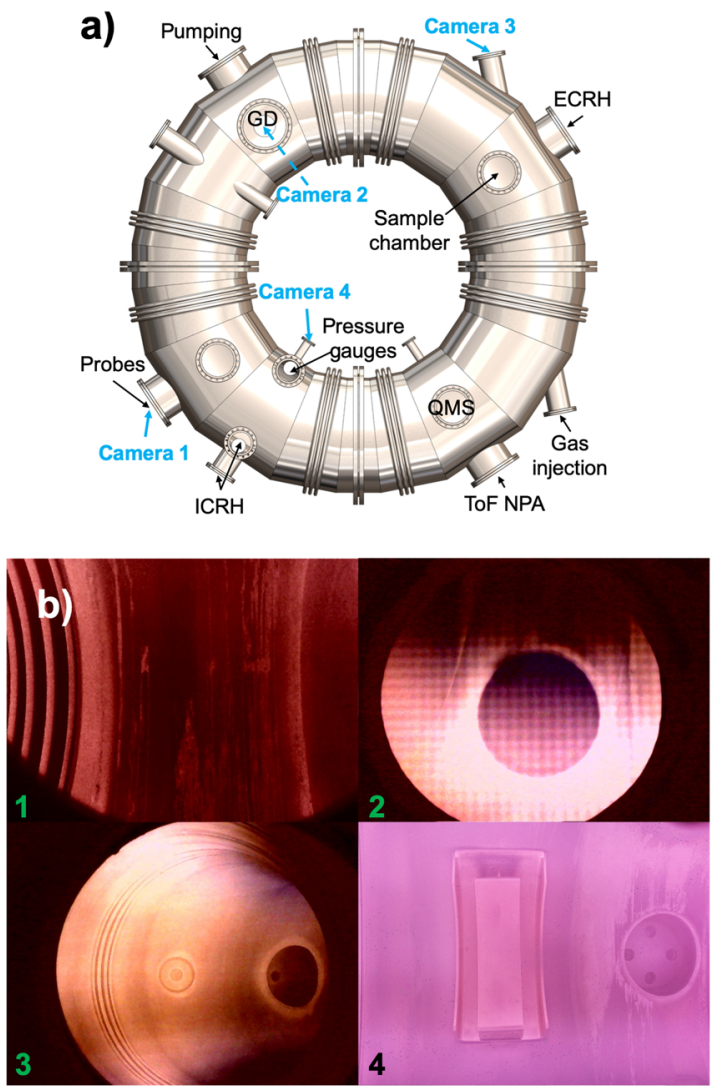

FIG. 19. (a) The scheme of the video diagnostic locations in TOMAS (Cameras 1 , 2, and 3 are Logitech C615 and Camera 4 is Arducam). (b) The combined view on helium ECRH plasma (pressure of $1.2 \times 10^{-4} \mathrm{mbar}$, input power of $1.5 \mathrm{~kW}$, and pulse length of $4 \mathrm{~s}$ ) from the location of 4 cameras [numbers on the frames correspond to camera numbers in (a).

Another video diagnostic is used for studies of ICRF plasma related events in the vicinity of the ICRF antenna. The video diagnostic in use is the Arducam 8MP wide angle drop-in replacement video camera for Raspberry Pi Camera Module V2. The camera module has an 8MP 1/4" SONY IMX219 CMOS image sensor. It is capable of taking $3280 \times 2464$ pixel static images and supports a maximum video resolution of $1920 \times 1080$ pixels with a frame rate of $30 \mathrm{fps}$. The camera module has a $175^{\circ}(\mathrm{D}) \times 145^{\circ}(\mathrm{H})$ $\times 77^{\circ}(\mathrm{W})$ wide-angle $\mathrm{M} 12$ lens. The lens has an effective focal length of $2.5 \mathrm{~mm}$ and an $\mathrm{f}$-number of 2.8 . The camera module is mounted inside an endoscope, exposed to atmospheric pressure, which is located in the body of the horizontal DN40 port in front of the ICRF antenna (Fig. 12). The plasma is observed through a $5 \mathrm{~mm}$ thick borosilicate glass mounted on a DN16 viewport. The distance between the lens and the inner wall of the vacuum vessel at the high field side is $\sim 10 \mathrm{~mm}$. Thus, the distance between the camera and the antenna strap is $480 \mathrm{~mm}$. The camera module can be rotated in the vertical plane. The module is connected by using a flexible cable to the Raspberry Pi Video Module V2.1 outside the endoscope. The software control of the video diagnostic is done by a small 
Raspberry PI 4 single-board computer. An example of the camera views is demonstrated in Fig. 19(b).

\section{The load-lock system for plasma surface interaction studies}

A new load-lock system with a vertical manipulator has been built for the exposure of material samples (i.e., probes) with geometrical parameters identical to those used in the neighboring linear plasma device PSI-2. ${ }^{26}$ The system allows probe exposures to a wide variety of plasma conditions at the desired radial distance from the plasma center and with a chosen surface orientation with respect to the magnetic field lines. The samples can be heated up to $600{ }^{\circ} \mathrm{C}$ to mimic exposure conditions of PFCs in fusion devices.

The system contains a sample chamber to enable exchange of probes. The chamber can be isolated from the TOMAS vacuum vessel by an electro-pneumatic gate valve. The sample chamber is installed on one of the vertical DN160 flanges, as shown in Fig. 20. The main element of the sample chamber is an expanded 4-way DN160 cross. The chamber is extended via one of the horizontal ports by the DN63 tee component. The extension is used to connect a Leybold PENNINGVAC PR27 pressure gauge $\left(1 \times 10^{-9} \mathrm{mbar}-\right.$ $1 \times 10^{-2}$ mbar) and an DN63 electro-pneumatic gate valve, which is further connected to the Pfeiffer Vacuum TSU 260 D turbomolecular pump system providing a maximum pumping speed of $210 \mathrm{l} / \mathrm{s}$ (for nitrogen/air) for $12.5 \mathrm{l}$ of the total volume of the chamber. The pumping station operates independent from the main vacuum system and is integrated in the control system (see Sec. III E). The opposite horizontal port of the cross is equipped by a DN160 quick-access

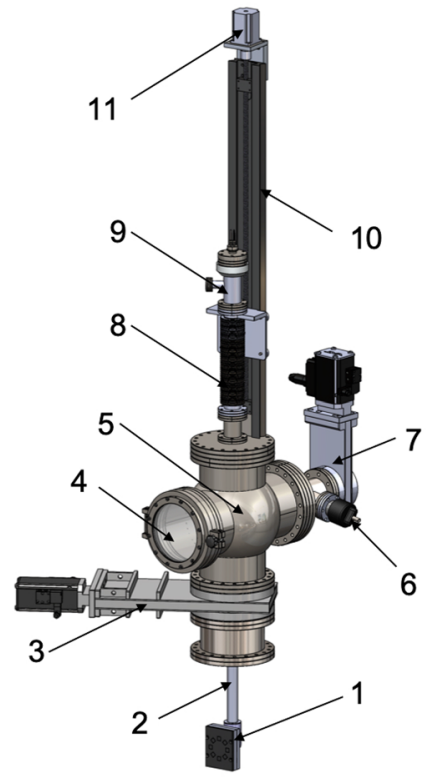

FIG. 20. The load-lock system. (1) PSI-2-like sample holder, (2) vacuum manipulator shaft, (3) DN160 gate valve, (4) DN160 quick-access door with a borosilicate sight glass, (5) sample chamber, (6) pressure gauge, (7) DN63 gate valve, (8) vacuum manipulator, (9) rotary feedthrough DN40, (10) manipulator base (rail), and (11) automated linear drive (step motor). door with a borosilicate sight glass, which is suitable for operation in pressure ranges down to $1 \times 10^{-8}$ mbar. It provides easy access for exchanging material samples and sample holder components inside the chamber. The top port of the cross is covered by a DN40 adapter flange whose horizontal surface serves as a base for a vertical vacuum manipulator (mounted on the DN40 connection) for insertion and positioning of probes in the TOMAS vessel. It is equipped by an automated linear drive that allows us to position samples with the precision of $\sim 1 \mathrm{~mm}$. The manipulator shaft with a diameter of $25.5 \mathrm{~mm}$ has a length of $1190 \mathrm{~mm}$, which is enough to move a sample holder down to the plasma center. The top end of the shaft is attached to a DN40 rotary feedthrough allowing angular positioning of the samples around the vertical axes with 360 degrees of freedom and an accuracy of $\pm 0.5^{\circ}$.

The sample holder itself consists of an adjustable adapter that fits to the manipulator shaft and a PSI-2 exchangeable target holder (Fig. 21). Both are made of stainless steel and connected to each other by two copper pins. The adapter allows us to tilt the holder with an angle of $0^{\circ}, 30^{\circ}, 45^{\circ}, 60^{\circ}$, or $90^{\circ}$, with respect to the vertical plane. The inclination angle can be set during the installation or exchange of material samples, while other types of sample positioning and orientation can be done even during plasma operation. The parts have a hollow structure to house thermocouples and electric wires and to reduce the heat transfer from the target holder to the manipulator tube.

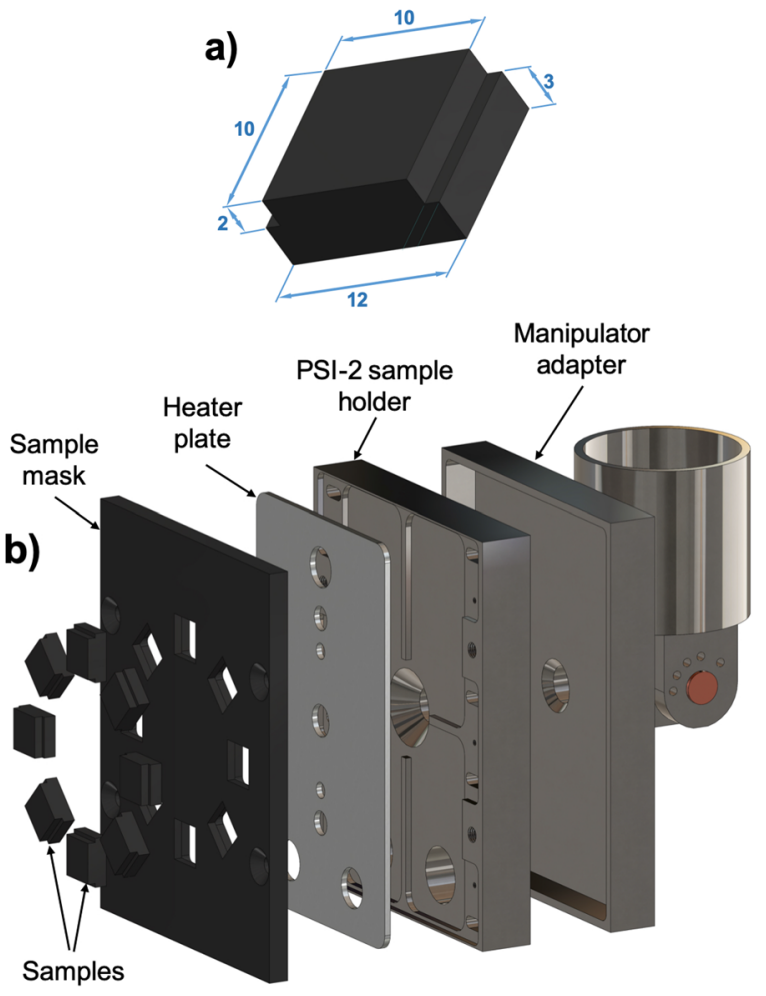

FIG. 21. (a) The sample probe (size and geometry). (b) The component mode of the sample holder (including the manipulator adapter, the sample holder, the heater plate, the sample mask with the samples). 
The PSI-2 target holders, which are presently used in TOMAS, consist of a holder base, an active heating element, and a mask (a fixing plate), which holds sample probes. The detailed model of the holder component is shown in Fig. 21. The stainless steel holder base has a rectangular profile with sides of $100 \mathrm{~mm}$ and $80 \mathrm{~mm}$. The active heating element made of a combination of a dielectric ceramic material (pyrolytic boron nitride) and an electrically conductive material (pyrolytic graphite) is located in the groove of the holder base. It allows us to achieve sample surface temperatures of up to $\sim 600^{\circ} \mathrm{C}$ without plasma exposure. To control the temperature of the material sample, a thermocouple (type $\mathrm{K}$ ) is connected to the inner mask surface. Copper wires with a diameter of $1.2 \mathrm{~mm}$ connect the heating element with a vacuum feedthrough from the vacuum on the manipulator top. Future upgrades may foresee active cooling of the holder for optimal feedback control on the sample temperature. Figure 21 includes an example of one of the different sample probe sizes and mask geometries available in PSI-2 and described in Ref. 26. The exposed surface of the sample probe has a square shape with a side of $10 \mathrm{~mm}$. Eight sample probes are located around the center of the mask in a circle with the diameter of $50 \mathrm{~mm}$. The total thickness of a sample has the same thickness of $5 \mathrm{~mm}$ as that for a sample mask. The masks are available in stainless steel, molybdenum, tungsten, and graphite.

\section{E. The control and data acquisition systems}

In the recent upgrade, TOMAS has been equipped by using an integrated control system (ICS) to connect and operate respective sub-systems. It also allows easy monitoring and provides safety interlocks. The control system of TOMAS is based on a CPU 15163PN/DP of the Siemens SIMATIC S7-1500 system. Additionally, the system contains two digital input signal modules (DI $32 \times 24 \mathrm{VDC}$ ), two digital output single modules (DQ $32 \times 24 \mathrm{VDC}$ ), two analog signal modules (AI $8 \times \mathrm{U} / \mathrm{I} / \mathrm{RTD} / \mathrm{TC}$ ), one analog signal module (AI $8 \times \mathrm{U} / \mathrm{I})$, and one analog output module $(\mathrm{AQ} 4 \times \mathrm{U} / \mathrm{I})$.

The control system is fully responsible for the operation of all pumping units (combinations of a rotary vane pre-pump and a turbopump) of TOMAS, including differential pumping of the diagnostics and gas evacuation (two rotary vane pumps) of the gas injection system. Moreover, all vacuum shutters (seven gate valves and two angle valves), separating different parts of the vessel and diagnostics, are run by the control system. Four pressure gauges (two Baratrons, one Pirani, and one Penning gauge) are integrated into the control system to monitor and record pressure in the vacuum vessel and the pumping duct. The main part of the gas injection system (3 mass flow controllers) is also operated via the control system with a safety interlock for hydrogen injection. Each of the four heating tape groups (four tapes in each group) of the baking system is individually controlled, simultaneously with temperature monitoring of the vacuum vessel segments. Additionally, the options to operate the glow discharge power supply and the automated tuning components of the RF matching system are added to the main control unit.

Besides the main part, which is located in a special rack, two separate Siemens ET200S periphery modules are located around TOMAS. One ET200S module is responsible for the control of the magnetic field coil power supply. It is placed in the rack of the power supply current rectifier and contains four digital input modules (DI
DC24V), one digital output modules (DO DC24V), two analog input modules (AI U), and two analog output modules (AO U). Another module, which is responsible for the control of the individual coil temperature and water flow in the primary cooling circuit (16 temperature gauges and 16 water flow monitors), is equipped with seven digital input modules (DI DC24V) and one digital output module (DO DC24V), and also 15 analog input modules (AI TC). The communication between all modules is provided via Siemens Profinet based on the Ethernet standard.

In order to avoid any critical conditions of the device operation, all systems can be de-energized immediately via an emergency shutdown provided by an Siemens Sirius 3 SK1 112 emergencystop relay. Currently, there are three emergency-stop switches on the machine (control rack, magnetic coil current rectifier, and glow discharge power supply). Each emergency-stop switch activates the relay, which is placed on the control rack. The relay has a separate $24 \mathrm{~V}$ power supply, allowing a safe power shut down. Load and control currents are split in the control system rack. Thus, in the case of emergency stop, only the load will be turned off and the SIMATIC control can keep operating.

The control system is equipped by using a panel personal computer (PC), installed in the main ICS rack and operated by Microsoft Windows, which makes permanent data log, storage of data, and system parameters in the database possible. It allows us to run and manipulate integrated sub-systems and display the current information about the TOMAS device and its system elements via user interface.

The currently used independent data acquisition system of TOMAS is based on 16 DEWETRON DAQP V high precision signal conditioning amplifiers with dynamic isolation. The modules have a bandwidth of $50 \mathrm{kHz}$ and an input range of $\pm 50 \mathrm{~V}$. The modules are connected to the National Instruments NI PXI-1042Q controller via two National Instruments TB-2709 terminal blocks. The data acquisition system is operated by LabView scripts.

\section{SUMMARY/CONCLUSION}

The upgraded TOMAS device is operated as a collaboration between IEK-4, Forschungszentrum Jülich, Germany; LPPERM/KMS, Brussels, Belgium; KTH, Stockholm, Sweden; and KIPT, Kharkov, Ukraine. The machine has been prepared for wall conditioning, plasma production, and plasma-surface interaction studies. The new auxiliary systems such as the pumping, gas injection, and pressure control systems and the improved magnetic field system together with the integrated control and data acquisition systems ensure the best up to date solutions facilitating the device operation. As demonstrated above, all plasma production systems, refurbished (ECRH) and newly installed (GD, ICRF), provide reliable operational results. The device is equipped with various diagnostics (pressure gauges, Langmuir probes, ToF NPA, QMS, and video cameras), allowing for the characterization of different plasma parameters. The load-lock system dedicated to PSI studies allows for exposures of various types of fusion-relevant materials to a wide variety of plasma conditions.

The first experimental sessions have shown that TOMAS achieves conditions that complement research in large fusion experiments. The upgraded device provides flexibility in operation under 
a broad range of conditions without time limits regarding the availability and access to the experiment. Besides the planned modernizations in the magnetic field power supply and completion of the control system, there are still opportunities for further upgrades and improvements to the existing components. The device is suitable for tests of new concepts in plasma production systems and development of specific diagnostics for EC/IC plasma studies in the toroidal magnetic field configuration. Therefore, the next step in operation will be dedicated to the full characterization of EC/IC plasmas at a wide range of operational parameters in order to continue the development of wall conditioning procedures and related aspects in plasma-wall interactions. Last but not least, TOMAS, being the "hands-on" machine, has become an excellent training tool for students and young researchers to obtain the necessary skills and experience in experimental physics.

\section{ACKNOWLEDGMENTS}

This work was carried out within the framework of the EUROfusion consortium and has received funding from the Euratom research and training programme 2014-2018 and 2019-2020 under Grant Agreement No. 633053. The views and opinions expressed herein do not necessarily reflect those of the European Commission.

\section{DATA AVAILABILITY}

The data that support the findings of this study are available from the corresponding author upon reasonable request.

\section{REFERENCES}

${ }^{1}$ G. Federici, C. H. Skinner, J. N. Brooks, J. P. Coad, C. Grisolia, A. A. Haasz, A. Hassanein, V. Philipps, C. S. Pitcher, J. Roth, W. R. Wampler, and D. G. Whyte, "Plasma-material interactions in current tokamaks and their implications for next step fusion reactors," Nucl. Fusion 41, 1967-2137 (2001).

${ }^{2}$ V. Philipps, P. Wienhold, A. Kirschner, and M. Rubel, "Erosion and redeposition of wall material in controlled fusion devices," Vacuum 67, 399-408 (2002).

${ }^{3}$ J. P. Coad, P. Andrew, D. E. Hole, S. Lehto, J. Likonen, G. F. Matthews, and M. Rubel, "Erosion/deposition in JET during the period 1999-2001," J. Nucl. Mater. 313-316, 419-423 (2003).

${ }^{4}$ T. Wauters, D. Borodin, R. Brakel, S. Brezinsek, K. J. Brunner, J. Buermans, S. Coda, A. Dinklage, D. Douai, O. Ford, G. Fuchert, A. Goriaev, H. Grote, A. Hakola, E. Joffrin, J. Knauer, T. Loarer, H. Laqua, A. Lyssoivan, V. Moiseenko, D. Moseev, J. Ongena, K. Rahbarnia, D. Ricci, V. Rohde, S. Romanelli, S. Sereda, T. Stange, F. L. Tabarés, L. Vanó, O. Volzke, and E. Wang, "Wall conditioning in fusion devices with superconducting coils," Plasma Phys. Controlled Fusion 62, $034002(2020)$

${ }^{5}$ H.-S. Bosch, R. Brakel, T. Braeuer, V. Bykov, P. Eeten, J. Feist, F. Füllenbach, M. Gasparotto, H. Grote, T. Klinger, H. Laqua, M. Nagel, D. Naujoks, M. Otte, K. Risse, T. Rummel, J. Schacht, A. Spring, T. Pedersen, and H. Trimiño Mora, "Final integration, commissioning and start of the Wendelstein 7-X stellarator operation," Nucl. Fusion 57, 16015 (2017).

${ }^{6}$ M. Shimada, D. J. Campbell, V. Mukhovatov, M. Fujiwara, N. Kirneva, K. Lackner, M. Nagami, V. D. Pustovitov, N. Uckan, J. Wesley, N. Asakura, A. E. Costley, A. J. H. Donné, E. J. Doyle, A. Fasoli, C. Gormezano, Y. Gribov, O. Gruber, T. C. Hender, W. Houlberg, S. Ide, Y. Kamada, A. Leonard,
B. Lipschultz, A. Loarte, K. Miyamoto, V. Mukhovatov, T. H. Osborne, A. Polevoi, and A. C. C. Sips, "ITER physics basis. Chapter 1: Overview and summary," Nucl. Fusion 47, S1-S17 (2007)

${ }^{7}$ H. B. Störk, J. Winter, J. Ihde, H. G. Esser, H. Reimer, and M. Freisinger, "TOMAS - A toroidal magnetized plasma facility for studying wall conditioning of future fusion devices," Fusion Sci. Technol. 39, 54-60 (2001).

${ }^{8}$ J. Ihde, H. B. Störk, J. Winter, M. Rubel, H. G. Esser, and H. Toyoda, "Wall conditioning by microwave generated plasmas in a toroidal magnetic field," J. Nucl. Mater. 290-293, 1180-1184 (2001).

${ }^{9}$ P. Sundelin, C. Schulz, V. Philipps, M. Rubel, G. Sergienko, and L. Marot, "Nitrogen-assisted removal of deuterated carbon layers," J. Nucl. Mater. 390-391, 647-650 (2009).

${ }^{10}$ C. Schulz, A. Kreter, V. Philipps, A. Litnovsky, and U. Samm, "Fuel removal from castellated structures by plasma discharges in hydrogen and oxygen," J. Nucl. Mater. 415, S781-S784 (2011).

${ }^{11}$ M. Ortner and L. G. Coliado Bandeira, "Magpylib: A free Python package for magnetic field computation," SoftwareX 11, 100466 (2020).

${ }^{12} \mathrm{~A}$. Goriaev, "First results of W7-X-Relevant conditioning procedures on the upgraded TOMAS device," in 44th EPS Conference on Plasma Physics, edited by M. Fajardo, E. Westerhof, C. Riconda, A. M. A. Bret, and B. Dromey (European Physical Society, 2017), Vol. 41F.

${ }^{13}$ A. Spring, R. Brakel, and H. Niedermeyer, "Wall conditioning for Wendelstein 7-X by glow discharge," Fusion Eng. Des. 66-68, 371-375 (2003).

${ }^{14}$ T. Härtl, A. Drenik, M. Kircher, V. Rohde, F. Stelzer, and W. Zeidner, "Optimization of the ASDEX upgrade glow discharge," Fusion Eng. Des. 124, 283-286 (2017).

${ }^{15}$ A. Spring, A. Cardella, B. Brucker, B. Mendelevitch, H. Niedermeyer, O. Sellmeier, S. Schweizer, and B. Streibl, "Design, manufacture and testing of the glow discharge electrodes for Wendelstein 7-X," Fusion Eng. Des. 86, 1933-1936 (2011).

${ }^{16}$ F. Louche, T. Wauters, R. Ragona, S. Möller, F. Durodié, A. Litnovsky, A. Lyssoivan, A. Messiaen, J. Ongena, P. Petersson, M. Rubel, S. Brezinsek, C. Linsmeier, and M. V. Schoor, "Design of an ICRF system for plasma-wall interactions and RF plasma production studies on TOMAS," Fusion Eng. Des. 123, 317-320 (2017).

${ }^{17}$ B. Schweer, J. Ongena, V. Borsuk, D. Birus, S. Bozhenkov, D. C. Bardawil, F. Durodié, D. Hartmann, K. P. Hollfeld, P. Kallmeyer, A. Krivska, F. Louche, A. Messiaen, O. Neubauer, G. Offermanns, G. Satheeswaran, M. Van Schoor, M. Vervier, and R. Wolf, "Development of an ICRH antenna system at W7-X for plasma heating and wall conditioning," Fusion Eng. Des. 123, 303-308 (2017).

${ }^{18} \mathrm{~F}$. Durodié and M. Vervier, "First results of the automatic matching device for TEXTOR'S ICRH system," in Fusion Technology 1992, edited by C. Ferro, M. Gasparotto, and H. Knoepfel (North-Holland, Oxford, 1993), pp. 477-480.

${ }^{19}$ F. Fasseur, M.S. thesis, ERM/KMS, 2017.

${ }^{20}$ K. Elinck, M.S. thesis, ERM/KMS, 2019.

${ }^{21} \mathrm{~S}$. L. Chen and T. Sekiguchi, "Instantaneous direct-display system of plasma parameters by means of triple probe," J. Appl. Phys. 36, 2363-2375 (1965).

${ }^{22}$ V. I. Demidov, S. V. Ratynskaia, and K. Rypdal, "Electric probes for plasmas: The link between theory and instrument," Rev. Sci. Instrum. 73, 3409-3439 (2002).

${ }^{23}$ D. López-Rodríguez, M.S. thesis, Ghent University, 2020.

${ }^{24}$ P. R. Brunsell, H. Bergsåker, M. Cecconello, J. R. Drake, R. M. Gravestijn, A. Hedqvist, and J.-A. Malmberg, "Initial results from the rebuilt EXTRAP T2R RFP device," Plasma Phys. Controlled Fusion 43, 1457-1470 (2001).

${ }^{25} \mathrm{M}$. Cecconello, "Neutral particle energy analyser based on time of flight technique for EXTRAP-T2R," Technical Report KTH/ALF/R-01/3-SE (KTH, Stockholm, Sweden, 2001).

${ }^{26}$ A. Kreter, C. Brandt, A. Huber, S. Kraus, S. Möller, M. Reinhart, B. Schweer, G. Sergienko, and B. Unterberg, "Linear plasma device PSI-2 for plasma-material interaction studies," Fusion Sci. Technol. 68, 8-14 (2015). 